\title{
Cubic Threefolds and Abelian Varieties of Dimension Five
}

\author{
Sebastian Casalaina-Martin* and Robert Friedman ${ }^{\dagger}$
}

November 28, 2018

\section{Introduction}

Cubic threefolds have been studied in algebraic geometry since classical times. In 4, Clemens and Griffiths proved the following beautiful result, which implies the irrationality of a smooth cubic threefold:

Theorem 1. Let $X$ be a smooth cubic threefold in $\mathbb{P}^{4}$ with intermediate Jacobian $J X$. Then the theta divisor of the 5-dimensional principally polarized abelian variety $J X$ has a unique singular point, which has multiplicity three.

In particular, the tangent cone to the theta divisor at the singular point is a cubic hypersurface in $\mathbb{P}^{4}$ which is projectively equivalent to $X$, thereby establishing the Torelli theorem for cubic threefolds.

Our goal in this paper is to prove a converse to the theorem of Clemens and Griffiths:

Theorem 2. Let $A$ be a principally polarized abelian variety of dimension 5. Suppose that the theta divisor $\Theta$ of $A$ has a unique singular point $p$, with mult $_{p} \Theta=3$. Then there exists a smooth cubic threefold $X$ such that $A \cong J X$ as principally polarized abelian varieties.

To prove Theorem 2] we use the well-known connection between $J X$ and Prym varieties, first established by Mumford [7]. Let $X$ be a smooth cubic threefold, and let $\lambda$ be a line contained in $X$. Projecting $X$ from $\lambda$ into $\mathbb{P}^{2}$ exhibits the blowup of $X$ along $\lambda$ as a conic bundle over $\mathbb{P}^{2}$, whose

${ }^{*}$ The first author was partially supported by a VIGRE fellowship from NSF grant DMS-98-10750.

${ }^{\dagger}$ The second author was partially supported by NSF grant DMS-02-00810. 
discriminant curve is a plane quintic $C$, smooth for a generic choice of $\lambda$, with an odd theta characteristic, corresponding to a double cover $\pi: \widetilde{C} \rightarrow C$. Moreover, $J X$ is isomorphic to the corresponding Prym variety $P(\widetilde{C}, \pi)$. From this, it is easy to determine the singularities of the theta divisor of $J X$.

To prove the converse, one has to be able to analyze the singularities of the theta divisor of an arbitrary principally polarized abelian variety of dimension 5. The general such abelian variety is the Prym variety of a smooth curve of genus 6 , and every principally polarized abelian variety of dimension 5 is the generalized Prym variety $P(\widetilde{C}, \pi)$ corresponding to an admissible double cover of a nodal curve in the sense of Beauville [1].

Thus the problem is connected to the problem of describing the singularities of the theta divisor of a Prym variety $P(\widetilde{C}, \pi)$. The set-theoretic description of these singularities goes back to Mumford 7 . The Prym variety $P(\widetilde{C}, \pi)=P$ may be viewed as a subvariety of the Jacobian $J \widetilde{C}$, and the intersection of the theta divisor $\widetilde{\Theta}$ of $J \widetilde{C}$ with $P$ is equal to $2 \Xi$, where $\Xi$ is a principal polarization on $P(\widetilde{C}, \pi)$. Thus a point $x \in P$ lies in the singular locus of $\Xi$ if and only if either mult $_{x} \widetilde{\Theta} \geq 4$, or mult $\widetilde{\Theta}_{\widetilde{\Theta}}=2$ but the tangent space to $P$ at $x$ is contained in the tangent cone to $\widetilde{\Theta}$ at $x$. These singularities are called stable and exceptional respectively. However, as pointed out by Smith and Varley [9, it is better to distinguish instead the two cases: (1) the tangent space to $P$ at $x$ is not contained in the tangent cone to $\widetilde{\Theta}$ at $x$ and (2) the tangent space to $P$ at $x$ is contained in the tangent cone to $\widetilde{\Theta}$ at $x$. In the first case, the multiplicity of $\Xi$ at $x$ is exactly one half the multiplicity of $\widetilde{\Theta}$ at $x$, whereas in the second case there is only an inequality. Thus one goal of this paper is to develop tools to handle the case (2). While we only discuss here some of the applications to cubic threefolds, the techniques of this paper can be developed much further. For example, one can prove directly that the multiplicity of the singular point of the theta divisor of $J X$ is three and use this to give another proof of the Torelli theorem for cubic threefolds, and it is possible to analyze the singularities of Prym theta divisors in many other situations. These applications will be discussed elsewhere, in the thesis of the first author.

The outline of the paper is as follows. Section 1 is a general discussion of how to calculate the multiplicity of points of Sing $\Xi$. The basic idea is to use test curves to determine the multiplicity, and to measure the multiplicity of $\Xi$ along such a curve by relating it to the extendability of sections to infinitesimal neighborhoods of a point. In Section 2, we deal with the case of a smooth curve $C$ and use the methods of Section 1 to show that, if $C$ is a smooth curve of genus 6 such that the theta divisor $\Xi$ of $P=P(\widetilde{C}, \pi)$ has a 
unique singular point of multiplicity 3 , then $C$ is a plane quintic. Roughly, the idea is as follows: if $L$ is the line bundle on $\widetilde{C}$ corresponding to the singular point, then $L$ is fixed by the involution $\tau$ on $\widetilde{C}$ corresponding to the double cover, and hence $L=\pi^{*} M$ for some line bundle $M$ on $C$, necessarily a theta characteristic. Moreover, Clifford's theorem implies that $h^{0}(L) \leq 6$, with equality only if $C$ is hyperelliptic (in which case the singular locus of $P$ is at least one-dimensional). The proof then involves analyzing the cases $h^{0}(L)=2$ and $h^{0}(L)=4$ and showing that a unique singular point $\Xi$ which is of multiplicity 3 can arise only for a plane quintic.

In Section 3, we extend the analysis to singular curves of genus 6 . The main difficulty arises from the fact that, in this case, a line bundle on $\widetilde{C}$ fixed by the involution is not necessarily the pullback of a line bundle from $C$. Nonetheless, such line bundles can be classified. Somewhat tedious arguments then lead to the same conclusion as for smooth curves, that $C$ is a plane quintic.

Finally, in Section 4, we discuss the proof of a result of Beauville, that the Prym variety of an admissible double cover of a plane quintic corresponding to an odd theta characteristic is in fact the intermediate Jacobian of a cubic threefold. This then completes the proof of Theorem 2

It is a pleasure to thank Arnaud Beauville and Roy Smith for discussions about their work on Prym varieties, plane quintics, and cubic threefolds.

\section{Theta divisors}

\subsection{Preliminaries}

We begin with an elementary lemma:

Lemma 1.1. Let $H$ be a hypersurface, not necessarily reduced, defined in a neighborhood of 0 in $\mathbb{C}^{n}$ and containing 0 . Let $f: \Delta \rightarrow \mathbb{C}^{n}$ be a holomorphic map, with $f(0)=0$. Then mult ${ }_{0} H \leq$ mult $_{0} f^{*} H$, and equality holds if and only if $f_{*}\left(T_{0} \Delta\right)$ is not contained in the tangent cone to $H$ at 0.

Let $C$ be a smooth projective curve of genus $g$, let $S$ be a scheme, and let $\mathcal{L}$ a line bundle over $C \times S$, of relative degree $g-1$. More generally, it suffices to assume that $C$ is an arbitrary projective curve and that $\chi\left(C ; \mathcal{L}_{s}\right)=0$ for every s. Then Mumford's construction [6, Theorem, p. 46] gives, locally

on $S$, a complex of locally free $\mathcal{O}_{S}$-modules $\mathcal{C}^{0} \stackrel{d}{\rightarrow} \mathcal{C}^{1}$ whose cohomology is $R^{0} \pi_{2 *} \mathcal{L}$ in dimension 0 and $R^{1} \pi_{2 *} \mathcal{L}$ in dimension 1 . Suppose that $\operatorname{det} d$ is not a zero divisor, and set $\Theta_{S}=(\operatorname{det} d)$. Then $\Theta_{S}$ is an effective nonzero 
Cartier divisor on $S$, which is independent of the choice of the complex $\mathcal{C}^{\bullet}$, and satisfies:

(i) The support of $\Theta_{S}$ is equal to the set of $s \in S$ such that $h^{0}\left(C ; \mathcal{L}_{s}\right) \neq 0$;

(ii) If $S=\operatorname{Pic}^{g-1} C$ and $\mathcal{L}$ is the Poincaré line bundle, then $\Theta_{S}$ is the usual theta divisor, with multiplicity one;

(iii) The construction is functorial: if $f: S^{\prime} \rightarrow S$ is a morphism, and $\mathcal{L}^{\prime}=$ $(\operatorname{Id} \times f)^{*} \mathcal{L}$, then $\Theta_{S^{\prime}}=f^{*} \Theta_{S}$.

(iv) If $S$ is smooth and $\operatorname{dim} S=1$, then $\Theta_{S}=\sum_{s \in S} \ell\left(\left(R^{1} \pi_{2 *} \mathcal{L}\right)_{s}\right) \cdot s$.

Clearly then we have the following, which for simplicity we shall just state in the case where $C$ is smooth:

Lemma 1.2. With notation as above, suppose $S$ is a smooth curve, that $f: S \rightarrow \mathrm{Pic}^{g-1} C$ is the morphism induced by the line bundle $\mathcal{L}$, and that $f\left(s_{0}\right)=x \in \mathrm{Pic}^{g-1} C$. Then mult ${ }_{x} \Theta \leq \operatorname{deg}_{s_{0}} \Theta_{S}=\ell\left(\left(R^{1} \pi_{2 *} \mathcal{L}\right)_{s_{0}}\right)$. Finally, there exists an $S$ and a line bundle $\mathcal{L}$ as above such that equality holds.

Let $L=\mathcal{L} \mid C \times\left\{s_{0}\right\}$. Since the map $\left(R^{1} \pi_{2 *} \mathcal{L}\right)_{s_{0}} \rightarrow H^{1}(C ; L)$ is surjective and there exists an $S$ of dimension one such that mult ${ }_{x} \Theta=\operatorname{deg}_{s_{0}} \Theta_{S}$, we see:

Lemma 1.3. If $L$ is the line bundle on $C$ corresponding to $x \in \mathrm{Pic}^{g-1} C$, then mult $_{x} \Theta \geq h^{1}(L)=h^{0}(L)$.

Our goal will eventually be to apply the above in the Prym case: Let $C$ be a smooth curve of genus $g, \pi: \widetilde{C} \rightarrow C$ a connected étale double cover. More generally, we will also consider admissible double covers in the sense of Beauville [1], and leave the necessary modifications to the reader. Let $P \subseteq J^{2 g-2} \widetilde{C}$ be the Prym variety: a line bundle $L$ on $\widetilde{C}$ belongs to $P$ if and only if $\operatorname{Norm}(L)=K_{C}$ and $h^{0}(L) \equiv 0 \bmod 2$. In this case, if $\widetilde{\Theta}$ is the canonical theta divisor of $J^{2 g-2} \widetilde{C}$, then $\widetilde{\Theta} \cap P=2 \Xi$, where $\Xi$ is the class of a principal polarization. Suppose that $S$ is a smooth curve with $s_{0} \in S$, and that $\mathcal{L}$ is a line bundle over $\widetilde{C} \times S$ of relative degree $2 g-2$ and such that the induced morphism $f: S \rightarrow J^{2 g-2} \widetilde{C}$ lies in $P$. For $x=f\left(s_{0}\right)$, we then have

Lemma 1.4. With notation as above,

$$
\frac{1}{2} h^{0}(L) \leq \operatorname{mult}_{x} \Xi \leq \frac{1}{2} \operatorname{deg}_{s_{0}} \Theta_{S}=\frac{1}{2} \ell\left(\left(R^{1} \pi_{2 *} \mathcal{L}\right)_{s_{0}}\right) .
$$

Moreover, there exists a choice of $S$ and a line bundle $\mathcal{L}$ as above such that $\operatorname{mult}_{x} \Xi=\frac{1}{2} \ell\left(\left(R^{1} \pi_{2 *} \mathcal{L}\right)_{s_{0}}\right)$. 


\subsection{Obstructions}

We return to the case of a general curve $C$ and theta divisor $\Theta$. Let $S$ be a smooth curve and let $t$ be a local coordinate for $S$ centered at $s_{0}$ and only vanishing there. Fix the following notation: $C_{k}=C \times \operatorname{Spec} \mathbb{C}[t] /\left(t^{k+1}\right)$, so that $C=C_{0}$, and $\mathcal{L}_{k}=\mathcal{L} / t^{k+1} \mathcal{L}$, so that $L=\mathcal{L}_{0}$. Thus $\mathcal{L}_{k}$ is the restriction of $\mathcal{L}$ to $C_{k}$, and $L$ is the restriction of $\mathcal{L}$ to $C \times\left\{s_{0}\right\}$. To calculate $\ell\left(\left(R^{1} \pi_{2 *} \mathcal{L}\right)_{s_{0}}\right)$, we use the following:

Lemma 1.5. For all $N$ sufficiently large, $\ell\left(H^{0}\left(C_{N} ; \mathcal{L}_{N}\right)\right.$ is independent of $N$ and is equal to $\ell\left(\left(R^{1} \pi_{2 *} \mathcal{L}\right)_{s_{0}}\right)$.

Proof. Choose $N$ so large that $t^{N+1}$ annihilates $\left(R^{1} \pi_{2 *} \mathcal{L}\right)_{s_{0}}$. Apply $R^{i} \pi_{2 *}$ to the short exact sequence

$$
0 \rightarrow \mathcal{L} \stackrel{t^{N+1}}{\longrightarrow} \mathcal{L} \rightarrow \mathcal{L}_{N} \rightarrow 0 .
$$

By assumption $R^{0} \pi_{2 *} \mathcal{L}=0$. Thus we obtain an exact sequence

$$
0=R^{0} \pi_{2 *} \mathcal{L} \rightarrow H^{0}\left(\mathcal{L}_{N}\right) \rightarrow R^{1} \pi_{2 *} \mathcal{L} \stackrel{t^{N+1}}{\rightarrow} R^{1} \pi_{2 *} \mathcal{L} .
$$

Since $R^{1} \pi_{2 *} \mathcal{L} \stackrel{t^{N+1}}{\longrightarrow} R^{1} \pi_{2 *} \mathcal{L}$ is the zero map, the homomorphism $H^{0}\left(\mathcal{L}_{N}\right) \rightarrow$ $R^{1} \pi_{2 *} \mathcal{L}$ is an isomorphism.

Let us be more explicit about the value of $N$ above. There is an exact sequence

$$
0 \rightarrow t \mathcal{L}_{k} \rightarrow \mathcal{L}_{k} \rightarrow L \rightarrow 0,
$$

where $t \mathcal{L}_{k} \cong \mathcal{L}_{k-1}$, and the obvious surjection $\mathcal{L}_{k} \rightarrow \mathcal{L}_{k-1}$ induces a commutative diagram

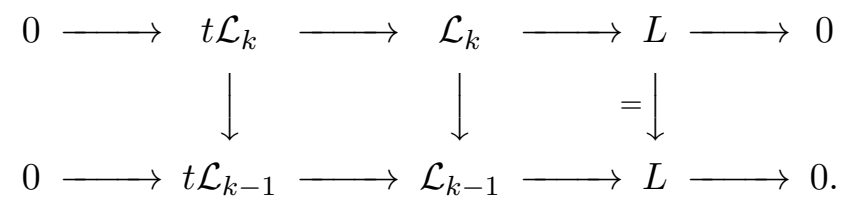

Let $\partial_{k}: H^{0}(L) \rightarrow H^{1}\left(t \mathcal{L}_{k}\right) \cong H^{1}\left(\mathcal{L}_{k-1}\right)$ be the coboundary. Then by naturality the image of $\partial_{k}$ in $H^{1}\left(\mathcal{L}_{k-2}\right)$ is equal to $\partial_{k-1}$. An easy induction then shows:

Lemma 1.6. Suppose in the above notation that $\partial_{N+1}$ is injective for some $N$. Then, for all $n \geq N$, the natural inclusion $t^{n-N} \mathcal{L}_{n} \subseteq \mathcal{L}_{n}$ induces an equality $H^{0}\left(t^{n-N} \mathcal{L}_{n}\right)=H^{0}\left(\mathcal{L}_{n}\right)$. In particular $\ell\left(H^{0}\left(\mathcal{L}_{n}\right)\right)=\ell\left(H^{0}\left(\mathcal{L}_{N}\right)\right)$ for all $n \geq N$. 
Suppose that $s \in H^{0}(L)$ lifts to a section $\tilde{s}$ of $H^{0}\left(\mathcal{L}_{k-1}\right)$, and hence that $\partial_{k-1}(s)=0$. Of course, we are free to vary $\tilde{s}$ by a section of $t \mathcal{L}_{k-1} \cong \mathcal{L}_{k-2}$. By the naturality statement above, the image of $\partial_{k}(s)$ in $H^{1}\left(\mathcal{L}_{k-2}\right)$ is equal to $\partial_{k-1}(s)=0$. It follows that $\partial_{k}(s)$ lies in the image of $H^{1}\left(t^{k-1} \mathcal{L}_{k-1}\right)$ in $H^{1}\left(\mathcal{L}_{k-1}\right)$, using the long exact sequence associated to

$$
0 \rightarrow t^{k-1} \mathcal{L}_{k-1} \rightarrow \mathcal{L}_{k-1} \rightarrow \mathcal{L}_{k-2} \rightarrow 0
$$

Thus, using the isomorphism $t^{k-1} \mathcal{L}_{k-1} \cong L$, there is a well-defined element $\gamma_{k}(s) \in H^{1}(L) / \operatorname{Im}\left(H^{0}\left(\mathcal{L}_{k-2}\right)\right)$ which represents the obstruction to lifting $s$ to a section of $\mathcal{L}_{k}$. Clearly, the image of $H^{0}\left(\mathcal{L}_{k-2}\right)$ represents the freedom to alter $\tilde{s}$ by a section of $t \mathcal{L}_{k-1} \cong \mathcal{L}_{k-2}$. In particular, if $\partial_{1}=\cdots=\partial_{k-1}=0$, then $\gamma_{k}(s)$ is a well-defined element of $H^{1}(L)$.

For $k=1$, we set $\gamma_{1}(s)=\partial_{1}(s)$. In this case, a well-known argument gives:

Lemma 1.7. Let $\xi \in H^{1}\left(\mathcal{O}_{C}\right)=\operatorname{Ext}^{1}(L, L)$ be the extension class corresponding to $\mathcal{L}_{1}$. Then $\partial_{1}(s)=s \cup \xi$, where the cup product is $H^{0}(L) \otimes$ $H^{1}\left(\mathcal{O}_{C}\right) \rightarrow H^{1}(L)$.

For computation, we will use the following which is an immediate consequence of the usual properties of cup product:

Lemma 1.8. Let $D$ be an effective divisor on $C$ and let $\partial: H^{0}\left(\mathcal{O}_{D}(D)\right) \rightarrow$ $H^{1}\left(\mathcal{O}_{C}\right)$ be the coboundary map induced by the short exact sequence

$$
0 \rightarrow \mathcal{O}_{C} \rightarrow \mathcal{O}_{C}(D) \rightarrow \mathcal{O}_{D}(D) \rightarrow 0
$$

Suppose that $\xi \in H^{1}\left(\mathcal{O}_{C}\right)$ is of the form $\partial(t)$ for some $t \in H^{0}\left(\mathcal{O}_{D}(D)\right)$. Then $s \cup \xi=\partial_{L}(s \cdot t)$, where $s \cdot t$ is the section of $L(D) \mid D$ given by taking the cup product of $s$ and $t$, and $\partial_{L}$ is the coboundary homomorphism arising from

$$
0 \rightarrow L \rightarrow L \otimes \mathcal{O}_{C}(D) \rightarrow L \otimes \mathcal{O}_{D}(D) \rightarrow 0 .
$$

As a consequence:

Theorem 1.9 (Riemann Singularity Theorem). If $L$ is the line bundle on $C$ corresponding to $x \in J^{g-1} C$, then mult $_{x} \Theta=h^{0}(L)$.

Proof. By Lemma 1.3 mult I $_{x} \Theta \geq h^{0}(L)$. To see the opposite inequality, suppose that we can find a $\xi \in H^{1}\left(\mathcal{O}_{C}\right)$ such that $\cdot \cup \xi: H^{0}(L) \rightarrow H^{1}(L)$ is injective. By Lemma 1.7 and Lemma 1.6, it follows that, given $S$ smooth of dimension one, $s_{0} \in S$, and $f: S \rightarrow \mathrm{Pic}^{g-1} C$ such that $f\left(s_{0}\right)=x$ and 
with tangent vector $\xi \operatorname{deg}\left(\Theta_{S}\right)_{s_{0}}=h^{0}(L)$. Since by Lemma 1.2 mult $_{x} \Theta \leq$ $\operatorname{deg}\left(\Theta_{S}\right)_{s_{0}}=h^{0}(L)$, we conclude that mult ${ }_{x} \Theta=h^{0}(L)$.

To find the appropriate $\xi$, apply Lemma 1.8 with $D=\sum_{i=1}^{r+1} p_{i}$, where $h^{1}(L)=r+1$ and the $p_{i}$ are general points of $C$, and $t$ is a section of $\mathcal{O}_{D}(D)$ which does not vanish at any of the $p_{i}$. If the $p_{i}$ are general, then $H^{0}\left(L \otimes \mathcal{O}_{C}(-D)\right)=0$. In particular $s \cdot t$ is nonzero for every nonzero $s \in H^{0}(L)$. It suffices to show that $\partial_{L}: H^{0}\left(L \otimes \mathcal{O}_{C}(D) \mid D\right) \rightarrow H^{1}(L)$ is injective, or equivalently that $H^{0}(L) \rightarrow H^{0}\left(L \otimes \mathcal{O}_{C}(D)\right)$ is surjective, i.e. that $\operatorname{dim} H^{0}\left(L \otimes \mathcal{O}_{C}(D)\right)=r+1$. By Riemann-Roch it suffices to show that $H^{1}\left(L \otimes \mathcal{O}_{C}(D)\right)=0$ or equivalently that $H^{0}\left(K_{C} \otimes L^{-1} \otimes \mathcal{O}_{C}(-D)\right)=0$. Since $h^{0}\left(K_{C} \otimes L^{-1}\right)=r+1$, if the $p_{i}$ are general then indeed $H^{0}\left(K_{C} \otimes\right.$ $\left.L^{-1} \otimes \mathcal{O}_{C}(-D)\right)=0$ and we are done.

\subsection{Cocycle calculations}

We conclude with some computations. For an appropriate affine cover $\left\{U_{i}\right\}$ of $C$, we may assume that $L$ has transition functions $\lambda_{i j}$ and that the transition functions for $\mathcal{L}$ are of the form $\lambda_{i j}(t)=\lambda_{i j}\left(1+\sum_{k=1}^{\infty} \alpha_{i j}^{(k)} t^{k}\right)$. Here $\xi=\alpha_{i j}^{(1)}$ is a 1-cocycle for $\mathcal{O}_{C}$. Likewise set

$$
\lambda_{i j ; N}(t)=\lambda_{i j}\left(1+\sum_{k=1}^{N} \alpha_{i j}^{(k)} t^{k}\right),
$$

a 1-cocycle for $\mathcal{O}_{C_{N}}^{*}$. Assume that $s \in H^{0}(L)$ and that $s_{N-1}$ is a lifting of $s$ to a section of $\mathcal{L}_{N-1}$. Thus using the trivialization over the open cover $\left\{U_{i}\right\}$ we have

$$
s_{i ; N-1}=s_{i}+\sum_{i=1}^{N-1} \sigma_{i}^{(k)} t^{k}
$$

for some functions $\sigma_{i}^{(k)}$ on $U_{i}$, with $s_{i ; N-1}=\lambda_{i j ; N-1}(t) s_{j ; N-1}$ on $\left(U_{i} \cap U_{j}\right) \times$ Spec $\mathbb{C}[t] /\left(t^{N}\right)$. Set $\sigma_{i}^{(0)}=s_{i}$. The section $s_{N-1}$ lifts to a section $s_{N}$ if and only if there exists $\sigma_{i}^{(N)} \in \Gamma \mathcal{O}_{U_{i}}$ such that, if we set $s_{i ; N}=s_{i}+\sum_{i=1}^{N} \sigma_{i}^{(k)} t^{k}$, then $s_{i ; N}=\lambda_{i j ; N}(t) s_{j ; N}$ on $\left(U_{i} \cap U_{j}\right) \times \operatorname{Spec} \mathbb{C}[t] /\left(t^{N+1}\right)$. Since $s_{i ; N-1}$ is already a section of $\mathcal{L}_{N-1}$, this becomes the equality

$$
\sigma_{i}^{(N)}=\lambda_{i j} \sigma_{j}^{(N)}+\sum_{k=0}^{N-1} \lambda_{i j} \alpha_{i j}^{(N-k)} \sigma_{j}^{(k)} .
$$

In other words, 
Lemma 1.10. The section $s_{N-1}$ lifts to a section $s_{N}$ if and only if the image of $\sum_{k=0}^{N-1} \lambda_{i j} \alpha_{i j}^{(N-k)} \sigma_{j}^{(k)}$ in $H^{1}(L)$ is zero. More generally, this image is the obstruction $\gamma_{k}(s)$ defined above.

Suppose that $p$ is a point of $C$, and fix once and for all a local coordinate $z$ at $p$. More precisely, let $\left\{U_{i}\right\}$ be an open cover of $C$, and assume that $p \in U_{0}$, that $p \notin U_{i}$ for $i \neq 0$, and that $z \in \Gamma \mathcal{O}_{U_{0}}$ is a coordinate centered at $p$. A calculation then shows:

Lemma 1.11. For $a \in \mathbb{C}$, let $\xi \in H^{1}\left(\mathcal{O}_{C}\right)$ be the image of $a / z$ under the coboundary map induced by the short exact sequence

$$
0 \rightarrow \mathcal{O}_{C} \rightarrow \mathcal{O}_{C}(p) \rightarrow \mathcal{O}_{C}(p) \mid p \rightarrow 0 .
$$

Let $s \in H^{0}(L)$ be a section such that $s(p)=0$. Then $s \cup \xi=0$ in $H^{1}(L)$, and in fact, choosing $\xi_{i j}$ to be given by the 1-cocycle

$$
\xi_{i j}= \begin{cases}a / z, & \text { if } i=0 \\ 0, & \text { otherwise }\end{cases}
$$

then $s \cup \xi=\delta \sigma$, where $\sigma$ is the 0-cochain defined by $\sigma_{0}=a s / z$ and $\sigma_{j}=0$ for $j \neq 0$, and $\delta$ is the Čech coboundary map.

We turn now to obstructions of order 2. More generally, consider the case where $S=C$, or an affine open subset of $C$ containing the point $p$, and $\mathcal{L}$ is either the line bundle $\pi_{1}^{*}\left(L \otimes \mathcal{O}_{C}(p)\right) \otimes \mathcal{O}_{C \times C}(-\Delta)$ or the line bundle $\pi_{1}^{*}\left(L \otimes \mathcal{O}_{C}(-p)\right) \otimes \mathcal{O}_{C \times C}(\Delta)$, where $\Delta \subseteq C \times C$ is the diagonal. We fix the coordinate $z$ as before, and let $t$ be the coordinate $z$, viewed as a coordinate on an affine open subset of $C$. In the first case, transition functions for $\mathcal{L}$ are of the form $\lambda_{i j}$, if neither $i$ nor $j$ is zero, whereas (for small $t$ ),

$$
\lambda_{0 j}(t)=\lambda_{0 j} \cdot\left(\frac{z-t}{z}\right)=\lambda_{0 j} \cdot\left(1-\left(\frac{t}{z}\right)\right) .
$$

In the second case, $\lambda_{i j}(t)=\lambda_{i j}$ if neither $i$ nor $j$ is zero, and (for small $t$ ),

$$
\lambda_{0 j}(t)=\lambda_{0 j} \cdot\left(\frac{z}{z-t}\right)=\lambda_{0 j} \cdot \sum_{k=0}^{\infty}\left(\frac{t^{k}}{z^{k}}\right) .
$$

In case $\mathcal{L}=\pi_{1}^{*}\left(L \otimes \mathcal{O}_{C}(p)\right) \otimes \mathcal{O}_{C \times C}(-\Delta)$, a first order lift corresponds to $\sigma_{i}^{(1)}$ satisfying

$$
\sigma_{0}^{(1)}=\lambda_{0 j} \sigma_{j}^{(1)}+\lambda_{0 j}\left(-\frac{1}{z}\right) s_{j}=\lambda_{0 j} \sigma_{j}^{(1)}-\frac{s_{0}}{z},
$$


and the second order obstruction satisfies

$$
\gamma_{2}(s)_{0 j}=\lambda_{0 j} \cdot 0 \cdot s_{j}+\lambda_{0 j}\left(-\frac{1}{z}\right) \sigma_{j}^{(1)}=-\frac{\sigma_{0}^{(1)}}{z}-\frac{s_{0}}{z^{2}} .
$$

Likewise, in case $\mathcal{L}=\pi_{1}^{*}\left(L \otimes \mathcal{O}_{C}(-p)\right) \otimes \mathcal{O}_{C \times C}(\Delta)$, a first order lift corresponds to $\sigma_{i}^{(1)}$ satisfying

$$
\sigma_{0}^{(1)}=\lambda_{0 j} \sigma_{j}^{(1)}+\lambda_{0 j}\left(\frac{1}{z}\right) s_{j}=\lambda_{0 j} \sigma_{j}^{(1)}+\frac{s_{0}}{z},
$$

and the second order obstruction satisfies

$$
\gamma_{2}(s)_{0 j}=\lambda_{0 j}\left(\frac{1}{z^{2}}\right) s_{j}+\lambda_{0 j}\left(\frac{1}{z}\right) \sigma_{j}^{(1)}=\frac{s_{0}}{z^{2}}+\left(\frac{\sigma_{0}^{(1)}}{z}-\frac{s_{0}}{z^{2}}\right)=\frac{\sigma_{0}^{(1)}}{z} .
$$

Lemma 1.12. With notation as above, suppose that $s \in H^{0}(L)$ lifts to a section of $\mathcal{L}_{1}$, corresponding to the 0 -cochain $\sigma_{i}^{(1)}$. Then $\gamma_{2}(s)=\left\{\gamma_{2}(s)_{i j}\right\}$ satisfies: $\gamma_{2}(s)_{i j}=0$ if neither $i$ nor $j$ is zero, and

$$
\gamma_{2}(s)_{0 j}= \begin{cases}-\sigma_{0}^{(1)} / z-s_{0} / z^{2}, & \text { if } \mathcal{L}=\pi_{1}^{*}\left(L \otimes \mathcal{O}_{C}(p)\right) \otimes \mathcal{O}_{C \times C}(-\Delta) ; \\ \sigma_{0}^{(1)} / z, & \text { if } \mathcal{L}=\pi_{1}^{*}\left(L \otimes \mathcal{O}_{C}(-p)\right) \otimes \mathcal{O}_{C \times C}(\Delta) .\end{cases}
$$

Proof. This follows from Lemma 1.10 and the above calculations.

An immediate calculation then gives:

Corollary 1.13. Let $\partial=\partial_{L, 2 p}$ be the coboundary map

$$
\partial: H^{0}\left(L \otimes \mathcal{O}_{C}(2 p) \mid 2 p\right) \rightarrow H^{1}(L)
$$

induced from the exact sequence

$$
0 \rightarrow L \rightarrow L \otimes \mathcal{O}_{C}(2 p) \rightarrow L \otimes \mathcal{O}_{C}(2 p) \mid 2 p \rightarrow 0,
$$

and let $a \in H^{0}\left(L \otimes \mathcal{O}_{C}(2 p) \mid 2 p\right)$ be defined by

$$
a= \begin{cases}\left.\left(-\frac{\sigma_{0}^{(1)}}{z}-\frac{s_{0}}{z^{2}}\right)\right|_{2 p}, & \text { if } \mathcal{L}=\pi_{1}^{*}\left(L \otimes \mathcal{O}_{C}(p)\right) \otimes \mathcal{O}_{C \times C}(-\Delta) ; \\ \left.\frac{\sigma_{0}^{(1)}}{z}\right|_{2 p}, & \text { if } \mathcal{L}=\pi_{1}^{*}\left(L \otimes \mathcal{O}_{C}(-p)\right) \otimes \mathcal{O}_{C \times C}(\Delta) .\end{cases}
$$

Then $\gamma_{2}(s)=\partial(a)$. 
Remark 1.14. We shall use the following two observations:

(i) The same calculations work for singular (reduced) curves $C$, provided that we use a smooth point $p \in C$.

(ii) The above calculations are local, in the sense that if

$$
\mathcal{L}=\mathcal{O}_{C}\left( \pm\left(p_{1}-q_{1}\right)\right) \otimes \cdots \otimes \mathcal{O}_{C}\left( \pm\left(p_{k}-q_{k}\right)\right),
$$

where $q_{i}=q_{i}(s)$ for some $s \in S$ with $q_{i}\left(s_{0}\right)=p_{i}$, then the local obstructions are sums of local contributions as calculated in the preceding lemmas.

\section{The Prym case}

We keep the following notation: $C$ is a smooth curve of genus $g$ with connected étale double cover $\pi: \widetilde{C} \rightarrow C$ and involution $\tau: \widetilde{C} \rightarrow \widetilde{C}$, and $\eta$ is the corresponding line bundle of order 2 on $C$. The Prym variety is denoted $P$ and its canonical theta divisor is $\Xi$.

\subsection{The balanced case}

We begin with a general criterion, along the lines of the Riemann singularity theorem, for when mult $_{x} \Xi=n$.

Lemma 2.1. Let $L$ be a line bundle on $\widetilde{C}$ corresponding to the point $x \in P$, and suppose that $h^{0}(L)=2 n$. Then mult $_{x} \Xi \geq \frac{1}{2} h^{0}(\widetilde{C} ; L)=n$. Moreover, if there exist distinct points $q_{1}, \ldots, q_{n} \in C$, with $\pi^{-1}\left(q_{i}\right)=\left\{p_{i}, \tau\left(p_{i}\right)\right\}$, such that the $2 n$ points $p_{1}, \tau\left(p_{1}\right), \ldots, p_{n}, \tau\left(p_{n}\right)$ impose independent conditions on $L$, then mult $_{x} \Xi=n$.

Proof. By Lemma 1.4 mult I $_{x} \Xi \geq \frac{1}{2} h^{0}(\widetilde{C} ; L)$. Moreover, equality holds if and only if mult ${ }_{x} \Xi \leq n$, if and only if there exists $\xi \in H^{1}\left(\mathcal{O}_{\widetilde{C}}\right)^{-}$such that the cup product map $\cup \xi: H^{0}(\widetilde{C} ; L) \rightarrow H^{1}(\widetilde{C} ; L)$ is injective.

Let $q_{1}, \ldots, q_{k}$ be points of $C$ and let $\pi^{-1}\left(q_{i}\right)=\left\{p_{i}, \tau\left(p_{i}\right)\right\}$. Set $D=$ $\sum_{i=1}^{k}\left(p_{i}+\tau\left(p_{i}\right)\right)$ and let $t$ be a section of $\mathcal{O}_{\widetilde{C}}(D) \mid D$ which is nonzero at each point in the support of $D$ and such that $\tau(t)=-t$. Let $\xi=\partial(t)$, so that $\tau^{*} \xi=-\xi$. The map $\cup \xi: H^{0}(L) \rightarrow H^{1}(L)$ is the composition of the maps $H^{0}(L) \rightarrow H^{0}\left(L \otimes \mathcal{O}_{\widetilde{C}}(D) \mid D\right)$ given by multiplying by $t$, and the coboundary $\partial_{L, D}: H^{0}\left(L \otimes \mathcal{O}_{\widetilde{C}}(D) \mid D\right) \rightarrow H^{1}(L)$ arising from the long exact sequence

$$
0 \rightarrow L \rightarrow L \otimes \mathcal{O}_{\widetilde{C}}(D) \rightarrow\left(L \otimes \mathcal{O}_{\widetilde{C}}(D)\right) \mid D \rightarrow 0 .
$$


Note that the map $s \in H^{0}(L) \mapsto s t$ is injective if and only if $H^{0}(L(-D))=0$, and $\partial_{L, D}$ is injective if and only if $H^{0}(L)=H^{0}(L(D))=2 n$. By RiemannRoch, $h^{0}(L(D))=h^{1}(L(D))+2 k$. Thus, if $\cup \xi$ fails to be injective, then either $h^{0}\left(L \otimes \mathcal{O}_{\widetilde{C}}(-D)\right) \neq 0$ or $h^{1}\left(L \otimes \mathcal{O}_{\widetilde{C}}(D)\right)>2 n-2 k$. Since by Serre duality $h^{1}\left(L \otimes \mathcal{O}_{\widetilde{C}}(D)\right)=h^{0}\left(\tau^{*} L \otimes \mathcal{O}_{\widetilde{C}}(-D)\right)=h^{0}\left(L \otimes \mathcal{O}_{\widetilde{C}}(-D)\right)$, for $k \leq n$ this last condition is equivalent to the condition that the $2 k$ points $p_{1}, \ldots, p_{k}, \tau\left(p_{1}\right), \ldots, \tau\left(p_{k}\right)$ fail to impose independent conditions on the free part of $L$. Likewise for $k=n, H^{0}\left(L \otimes \mathcal{O}_{\widetilde{C}}(-D)\right) \neq 0$ if and only if the $2 n$ points $p_{1}, \ldots, p_{n}, \tau\left(p_{1}\right), \ldots, \tau\left(p_{n}\right)$ fail to impose independent conditions on the free part of $L$. Hence, if the points $p_{1}, \tau\left(p_{1}\right), \ldots, p_{n}, \tau\left(p_{n}\right)$ impose independent conditions on $L$, then $\cup \xi$ is injective and hence mult $x_{x} \Xi=n$.

Remark 2.2. An elementary argument shows the following result of SmithVarley [9]: if $h^{0}(L)=2 n$ but, for every choice of distinct points $q_{1}, \ldots, q_{n} \in$ $C$ with $\pi^{-1}\left(q_{i}\right)=\left\{p_{i}, \tau\left(p_{i}\right)\right\}$, the $2 n$ points $p_{1}, \tau\left(p_{1}\right), \ldots, p_{n}, \tau\left(p_{n}\right)$ fail to impose independent conditions on $L$, then $L$ is of the form $\pi^{*} M(E)$, where $M$ is a line bundle on $C$ with $h^{0}(M) \geq 2$ and $E$ is effective.

Theorem 2.3 (Smith-Varley $[\mathbf{9}]$ ). Let $L$ be a line bundle on $\widetilde{C}$ corresponding to the point $x \in P$. Suppose that $L=\pi^{*} M \otimes \mathcal{O}_{\widetilde{C}}(B)$, where $h^{0}(L)=h^{0}\left(\pi^{*} M\right)$, so that $B$ is contained in the base locus of $L$. If $h^{0}(M)=$ $h^{0}(M \otimes \eta)$, then mult $_{x} \Xi=n$. If $h^{0}(M) \neq h^{0}(M \otimes \eta)$, then, for every line bundle $\mathcal{L}_{1}$ over $C \times \operatorname{Spec} \mathbb{C}[t] /\left(t^{2}\right)$ restricting to $L$ on the closed fiber and corresponding to a morphism Spec $\mathbb{C}[t] /\left(t^{2}\right) \rightarrow P, \ell\left(h^{0}\left(\mathcal{L}_{1}\right)\right) \geq$ $2 \max \left(h^{0}(M), h^{0}(M \otimes \eta)\right)$. Hence

$$
\operatorname{mult}_{x} \Xi \geq \max \left(h^{0}(M), h^{0}(M \otimes \eta)\right)>\frac{1}{2} h^{0}(L) .
$$

Proof. By assumption, $L=\pi^{*} M \otimes \mathcal{O}_{\widetilde{C}}(B)$, where $h^{0}(L)=h^{0}\left(\pi^{*} M\right)$. First suppose that $h^{0}(M)=h^{0}(M \otimes \eta)=n$. Note that

$$
K_{\widetilde{C}} \otimes L^{-1}=\tau^{*} L=\pi^{*} M \otimes \mathcal{O}_{\widetilde{C}}(\tau(B)) .
$$

Thus $h^{0}\left(K_{\widetilde{C}} \otimes L^{-1}\right)=h^{0}\left(\pi^{*} M\right)$ as well. Choose $n$ general points $q_{1}, \ldots, q_{n}$ on $C$ and write $\pi^{-1}\left(q_{i}\right)=\left\{p_{i}, \tau\left(p_{i}\right)\right\}$. Let $D=\sum_{i=1}^{n}\left(p_{i}+\tau\left(p_{i}\right)\right)$, let $t$ be a section of $\mathcal{O}_{\widetilde{C}}(D) \mid D$ which is nonzero at each point in the support of $D$ and such that $\tau(t)=-t$, and let $\partial(t)=\xi$. It suffices to find $q_{i}$ so that

$$
H^{0}\left(L \otimes \mathcal{O}_{\widetilde{C}}(-D)\right)=H^{1}\left(L \otimes \mathcal{O}_{\widetilde{C}}(D)\right)=0 .
$$


We have seen in the course of the proof of Lemma 2.1 that both groups have the same dimension. Working for example with $H^{0}\left(L \otimes \mathcal{O}_{\widetilde{C}}(-D)\right)$, we have

$$
\begin{aligned}
h^{0}\left(L \otimes \mathcal{O}_{\widetilde{C}}(-D)\right) & =h^{0}\left(\pi^{*}\left(M \otimes \mathcal{O}_{C}\left(-\sum_{i} q_{i}\right)\right)\right) \\
& =h^{0}\left(M \otimes \mathcal{O}_{C}\left(-\sum_{i} q_{i}\right)\right)+h^{0}\left(M \otimes \eta \otimes \mathcal{O}_{C}\left(-\sum_{i} q_{i}\right)\right) .
\end{aligned}
$$

Since $h^{0}(M)=h^{0}(M \otimes \eta)=n$, if the $q_{i}$ are general then both of the terms in the last line are zero as claimed. Hence $H^{0}\left(L \otimes \mathcal{O}_{\widetilde{C}}(-D)\right)=$ $H^{1}\left(L \otimes \mathcal{O}_{\widetilde{C}}(D)\right)=0$.

Finally assume that $L=\pi^{*} M(B)$, with $h^{0}(M)=n_{1}>h^{0}(M \otimes \eta)=n_{2}$. For $\xi \in H^{1}\left(\mathcal{O}_{\widetilde{C}}\right)^{-}$, consider the pairing $H^{0}(L) \otimes H^{0}\left(\tau^{*} L\right) \rightarrow \mathbb{C}$ defined by

$$
\langle s, t\rangle=(s t) \cup \xi \in H^{1}\left(L \otimes \tau^{*} L\right)=H^{1}\left(K_{\widetilde{C}}\right) \cong \mathbb{C} .
$$

Since $\tau$ acts trivially on $H^{1}\left(K_{\widetilde{C}}\right)$, we must have $\langle\tau(t), \tau(s)\rangle=-\langle s, t\rangle$. By Serre duality, it suffices to show that $\langle\cdot, \cdot\rangle$ is degenerate in the first variable for every $\xi$. But $H^{0}(L)=H^{0}\left(\pi^{*} M\right)$ and $H^{0}\left(\tau^{*} L\right)=H^{0}\left(\pi^{*} M(\tau(B))\right)=$ $H^{0}\left(\pi^{*} M\right)$, so that $\langle\cdot, \cdot\rangle$ induces a pairing $H^{0}\left(\pi^{*} M\right) \otimes H^{0}\left(\pi^{*} M\right) \rightarrow \mathbb{C}$. Clearly, given sections $s, t \in H^{0}\left(\pi^{*} M\right),\langle i(s), i(t)\rangle=i(s t) \cup \xi$, where we denote by $i$ any of the natural inclusions $\pi^{*} M \rightarrow \pi^{*} M(B), \pi^{*} M \rightarrow \pi^{*} M(\tau(B)$ ), or $\pi^{*} M^{\otimes 2} \rightarrow \pi^{*} M(B) \otimes \pi^{*} M(\tau(B))$, and thus $\langle i(s), i(t)\rangle=\langle i(t), i(s)\rangle$ for all $s, t \in H^{0}\left(\pi^{*} M\right)$. Hence, for $s, t \in H^{0}\left(\pi^{*} M\right) \otimes H^{0}\left(\pi^{*} M\right),\langle\tau(i(s)), \tau(i(t))\rangle=$ $-\langle i(s), i(t)\rangle$. It follows that $\langle\cdot, \cdot\rangle$ pairs $H^{0}(M)$ with $H^{0}(M \otimes \eta)$ and in fact has a kernel of dimension at least $n_{1}-n_{2}$. Hence $\cup \xi$ has a kernel of dimension at least $n_{1}-n_{2}$. Thus, if $\mathcal{L}_{1}$ is the line bundle over $C \times \operatorname{Spec} \mathbb{C}[t] /\left(t^{2}\right)$ corresponding to $\xi, \ell\left(\mathcal{L}_{1}\right) \geq\left(n_{1}+n_{2}\right)+\left(n_{1}-n_{2}\right)=2 n_{1}$. The final statement of Theorem 2.3 is then clear.

\subsection{The case where all sections are invariant}

We consider now the opposite extreme, where all of $H^{0}(L)$ is invariant. Our goal is to show:

Theorem 2.4. Suppose that $L=\pi^{*} M$ corresponds to the point $x \in P$ and that $h^{0}(L)=h^{0}(M)=2 n>0$. Then mult $_{x} \Xi=2 n$.

Proof. Since $L \in P$, Norm $L=M^{\otimes 2}=K_{C}$. Thus $M$ and $L$ are theta characteristics.

Next we show that every section of $L$ lifts to first order: 
Lemma 2.5. Suppose that $L=\pi^{*} M$ is as above and that $h^{0}(L)=h^{0}(M)$. Let $\mathcal{L}_{1}$ be a line bundle over $C \times \operatorname{Spec} \mathbb{C}[t] /\left(t^{2}\right)$ restricting to $L$ on the closed fiber and corresponding to a morphism $\mathrm{Spec} \mathbb{C}[t] /\left(t^{2}\right) \rightarrow P$. Then the homomorphism $H^{0}\left(\mathcal{L}_{1}\right) \rightarrow H^{0}(L)$ is surjective, and hence $\ell\left(H^{0}\left(\mathcal{L}_{1}\right)\right)=2 h^{0}(L)$ and $\operatorname{mult}_{x} \Xi \geq 2 n$.

Proof. By Theorem 2.3 and its proof, the homomorphism $\cup \xi: H^{0}(L) \rightarrow$ $H^{1}(L)$ is zero for every $\xi \in H^{1}\left(\mathcal{O}_{\widetilde{C}}\right)^{-}$. Thus $H^{0}\left(\mathcal{L}_{1}\right) \rightarrow H^{0}(L)$ is surjective and $\ell\left(H^{0}\left(\mathcal{L}_{1}\right)\right)=2 h^{0}(L)$. The last statement is clear.

Lemma 2.6. With assumptions as above, suppose that $h^{0}(L)=2 n$ and that $q_{1}, \ldots, q_{n}$ are general points of $C$ with $\pi^{-1}\left(q_{i}\right)=\left\{p_{i}, \tau\left(p_{i}\right)\right\}$. Let $D=$ $\sum_{i}\left(p_{i}+\tau\left(p_{i}\right)\right)$. Then the natural inclusion $H^{0}(L) \rightarrow H^{0}\left(L \otimes \mathcal{O}_{\widetilde{C}}(2 D)\right)^{+}$is an isomorphism.

Proof. It suffices to show that $\operatorname{dim} H^{0}\left(L \otimes \mathcal{O}_{\widetilde{C}}(2 D)\right)^{+}=2 n$. Now

$$
H^{0}\left(L \otimes \mathcal{O}_{\widetilde{C}}(2 D)\right)^{+}=H^{0}\left(M \otimes \mathcal{O}_{C}\left(2 \sum_{i} q_{i}\right)\right) .
$$

By Riemann-Roch, $h^{0}\left(M \otimes \mathcal{O}_{C}\left(2 \sum_{i} q_{i}\right)\right)=2 n+h^{1}\left(M \otimes \mathcal{O}_{C}\left(2 \sum_{i} q_{i}\right)\right)$. So it suffices to show that $h^{1}\left(M \otimes \mathcal{O}_{C}\left(2 \sum_{i} q_{i}\right)\right)=0$. Since $M$ is a theta characteristic, it suffices to show that $h^{0}\left(M \otimes \mathcal{O}_{C}\left(-2 \sum_{i} q_{i}\right)\right)=0$. Clearly this holds if the $q_{i}$ are generic.

Corollary 2.7. With notation and assumptions as above, let $D=\sum_{i}\left(p_{i}+\right.$ $\left.\tau\left(p_{i}\right)\right)$ and let $\partial_{L, 2 D}: H^{0}\left(L \otimes \mathcal{O}_{\widetilde{C}}(2 D) \mid 2 D\right) \rightarrow H^{1}(L)$ be the coboundary map induced by the long exact sequence

$$
0 \rightarrow L \rightarrow L \otimes \mathcal{O}_{\widetilde{C}}(2 D) \rightarrow L \otimes \mathcal{O}_{\widetilde{C}}(2 D) \mid 2 D \rightarrow 0 .
$$

Then $\operatorname{Ker} \partial_{L, 2 D}$ is $\tau$-anti-invariant.

Proof. The long exact cohomology sequence is acted on by $\tau$ and gives a long exact sequence of $\tau$-invariants. In particular, $\left(\operatorname{Ker} \partial_{L, D}\right)^{+}$is the image of $H^{0}\left(L \otimes \mathcal{O}_{\widetilde{C}}\left(2 \sum_{i}\left(p_{i}+\tau\left(p_{i}\right)\right)\right)^{+} / H^{0}(L)^{+}\right.$. By the previous lemma, this group is zero.

We now complete the proof of Theorem 2.4. Choose $n$ general points $q_{i}$ of $C$ and set $\pi^{-1}\left(q_{i}\right)=\left\{p_{i}, \tau\left(p_{i}\right)\right\}, D=\sum_{i=1}^{n}\left(p_{i}+\tau\left(p_{i}\right)\right)$. Let

$$
\mathcal{L}_{t}=L \otimes \mathcal{O}_{\widetilde{C}}\left(\sum_{i=1}^{n}\left(p_{i}-\tau\left(p_{i}\right)-r_{i}(t)+\tau\left(r_{i}(t)\right)\right)\right)
$$


be a line bundle over $C \times \Delta$, where $\Delta$ is the disk and the $r_{i}: \Delta \rightarrow C$ are isomorphisms from $\Delta$ to a neighborhood of $p_{i}$ such that $r_{i}(0)=p_{i}$. We have seen that every section $s$ of $L$ lifts to first order. Thus it suffices to show that $\gamma_{2}(s) \neq 0$ for every nonzero section $s$. Here $\gamma_{2}(s)$ is a well-defined element of $H^{1}(L)$, given by Lemma 1.12 By Corollary 1.13, if $\gamma_{2}(s)=0$, then $\partial_{L, 2 D}(a)=0$, where $a \in H^{0}\left(L \otimes \mathcal{O}_{\widetilde{C}}(2 D) \mid 2 D\right)$ given as follows: if $\xi \in H^{1}\left(\mathcal{O}_{\widetilde{C}}\right)$ is the class corresponding to the first order deformation defined by $\mathcal{L}$ and $s \cup \xi=\delta\left(\sigma^{(1)}\right)$, then, for a local $\tau$-invariant coordinate $z_{i}$ around $p_{i}$ and $\tau\left(p_{i}\right)$, we have

$$
a_{p_{i}}=\left.\left(-\frac{\sigma_{0}^{(1)}}{z_{i}}-\frac{s}{z_{i}^{2}}\right)\right|_{2 p} ; \quad a_{\tau\left(p_{i}\right)}=\left.\frac{\sigma_{0}^{(1)}}{z_{i}}\right|_{2 p} .
$$

By Corollary 2.7, the class $a$ is $\tau$-anti-invariant. Clearly, this is only possible if $s_{0}\left(p_{i}\right)=0$ for every $i$, and hence $s_{0}\left(\tau\left(p_{i}\right)\right)=0$ as well by invariance. In this case, Lemma 1.11 implies that we can take $\sigma^{(1)}$ to be as follows: $\sigma_{p_{i}}^{(1)}=$ $-s / z_{i}$, and $\sigma_{\tau\left(p_{i}\right)}^{(1)}=s / z_{i}$. Thus $a_{p_{i}}=-2 s / z_{i}^{2}$ and $a_{\tau\left(p_{i}\right)}=s / z_{i}^{2}$. Since $s$ is $\tau$-invariant, $a$ is $\tau$-anti-invariant if and only if $s$ vanishes to order 2 at $p_{i}$ and $\tau\left(p_{i}\right)$ for every $i$. But clearly if the points $q_{i}$ are chosen generically as in the proof of Lemma 2.6 then $H^{0}\left(L \otimes \mathcal{O}_{\widetilde{C}}(-2 D)\right)=H^{0}\left(M \otimes \mathcal{O}_{C}\left(-2 \sum_{i=1}^{n} q_{i}\right)\right)=$ 0 . Thus $\gamma_{2}(s)=0$ if and only if $s=0$. It follows that, for every $N \geq$ $2, h^{0}\left(\mathcal{L}_{N}\right)=2 n$ and so mult $x \Xi \leq 2 n$. Since we have already seen that mult $_{x} \Xi \geq 2 n$, equality must hold. This completes the proof of Theorem 2.4

We can now prove the main theorem for Prym varieties of smooth curves of genus 6 .

Theorem 2.8. Let $\pi: \widetilde{C} \rightarrow C$ be a connected étale double cover of a smooth curve of genus 6 . If the theta divisor of the associated Prym variety $P(\widetilde{C}, \pi)$ has a unique singular point which is an isolated triple point, then $C$ is a plane quintic and $\pi: \widetilde{C} \rightarrow C$ is the double cover associated to an odd theta characteristic.

Proof. Let $L$ be a line bundle on $\widetilde{C}$ of degree 10 corresponding to a singular point of the theta divisor $\Xi$ of $P(\widetilde{C}, \pi)$. In particular, $h^{0}(L)=2 n>0$. By Clifford's theorem, $h^{0}(L) \leq 6$, with equality if and only if $\widetilde{C}$ is hyperelliptic. This easily implies that $C$ is hyperelliptic (see below for the singular case), and then $\Xi$ does not have an isolated singularity. Thus we may assume that $h^{0}(L)<6$, and hence $h^{0}(L) \leq 4$. 
Since there is a unique singular point of $\Xi, \tau^{*} L=L$, and by étale descent $L=\pi^{*} M$. Thus $h^{0}(L)=h^{0}(M)+h^{0}(M \otimes \eta) \leq 4$, where $\eta$ is the line bundle of order two corresponding to $\widetilde{C}$. We may assume that $h^{0}(M) \geq h^{0}(M \otimes \eta)$ and that $h^{0}(M) \neq 0$. By Clifford's theorem, $h^{0}(M) \leq 5 / 2+1<4$. If $h^{0}(M)=1$, then the only possibility is $h^{0}(M \otimes \eta)=1$ as well. In this case the multiplicity of the corresponding point of $\Xi$ is $h^{0}(M)=1$, by Theorem 2.3 . If $h^{0}(M)=2=h^{0}(M \otimes \eta)$, then the multiplicity of the corresponding point of $\Xi$ is $h^{0}(M)=2$, again by Theorem 2.3. If $h^{0}(M)=2$ and $h^{0}(M \otimes \eta)<2$, then $h^{0}(M \otimes \eta)=0$. By Theorem 2.4 the multiplicity of the singular point of $\Xi$ is 2 in this case as well.

Suppose finally that $h^{0}(M)=3$. Then $h^{0}(M \otimes \eta)=1$. If $M$ has a base point, then $C$ is hyperelliptic, again by Clifford's theorem, and so $\Xi$ does not have an isolated singularity. Otherwise, $M$ defines a morphism from $C$ to $\mathbb{P}^{2}$, which is birational since $M$ has prime degree and is an embedding since the genus of a smooth plane quintic is 6 . Thus the only case that can arise is: $C$ is a smooth plane quintic, $h^{0}(M)=3$ and $M$ is the pullback of $\mathcal{O}_{\mathbb{P}^{2}}(1)$, and $h^{0}(M \otimes \eta)=1$. This concludes the proof.

\section{Singular curves}

\subsection{Preliminaries}

We extend the results of the previous section to the case of singular curves. Let $\mathcal{R}_{g}$ denote the space of admissible covers constructed in [1], such that the associated Prym morphism $P: \mathcal{R}_{g} \rightarrow \mathcal{A}_{g-1}$ is defined, dominant, and proper, and in particular it is surjective. A point of $\mathcal{R}_{g}$ will be denoted by $(\widetilde{C}, \pi)$, with corresponding involution $\tau$, and the corresponding Prym variety by $P(\widetilde{C}, \pi)$. Since we are only concerned with principally polarized abelian varieties whose theta divisors have an isolated singular point, and hence are irreducible, we will always assume that $(\widetilde{C}, \pi)$ satisfies condition $(*)$ of Beauville: the fixed points of $\tau$ are exactly the singular points of $\widetilde{C}$, and at a fixed point the local branches of $\widetilde{C}$ are not exchanged. (Otherwise by [1, Theorem 5.4], $P(\widetilde{C}, \pi)$ is either reducible or a Jacobian.) Moreover, we can also assume that, if there is a decomposition $\widetilde{C}=\widetilde{C}_{1} \cup \widetilde{C}_{2}$, with $\widetilde{C}_{1} \cap \widetilde{C}_{2}$ finite, then $\#\left(\widetilde{C}_{1} \cap \widetilde{C}_{2}\right)$ is even and $\geq 4$ since if $\#\left(\widetilde{C}_{1} \cap \widetilde{C}_{2}\right)=2$, then, by 1. Lemma 4.11], $P(\widetilde{C}, \pi)$ is reducible. Of course, since the components and double points of $\widetilde{C}$ are identified with those of $C$, a similar statement holds for $C$.

Let $L$ be a line bundle on $\widetilde{C}$ such that Norm $L=\omega_{C}$, and hence $L \otimes \tau^{*} L=$ $\omega_{\widetilde{C}}$. We will need the following numerology: 
Lemma 3.1. Let $L$ be a line bundle on $\widetilde{C}$ such that $\operatorname{Norm} L=\omega_{C}$, and hence $L \otimes \tau^{*} L=\omega_{\widetilde{C}}$. Suppose that the irreducible components of $\widetilde{C}$ are $C_{i}, \ldots, C_{k}$, with $p_{a}\left(C_{i}\right)=p_{i}$. Suppose that $\bar{C}_{i}$ is the component of $C$ lying under $C_{i}$, with $p_{a}\left(\bar{C}_{i}\right)=\bar{p}_{i}$. Let $b_{i}=\#\left(C_{i} \cap\left(\bigcup_{j \neq i} C_{j}\right)\right)$. Then

$$
\operatorname{deg}\left(L \mid C_{i}\right)=p_{i}-1+\frac{b_{i}}{2}=2 \bar{p}_{i}-2+b_{i} .
$$

In particular $\operatorname{deg}\left(L \mid C_{i}\right)$ is even and, as $b_{i} \geq 4 \operatorname{deg}\left(L \mid C_{i}\right) \geq 2 \bar{p}_{i}+2 \geq 2$. Finally, $p_{i} \geq 1$, since $C_{i} \rightarrow \bar{C}_{i}$ is a double cover with at least 4 branch points.

\subsection{Clifford's theorem and hyperelliptic curves}

Definition 3.2. Let $C$ be a nodal curve. A $g_{2}^{1}$ on $C$ is a line bundle $L$ such that $\operatorname{deg} L=2$ and $h^{0}(C ; L)=2$. We call $L$ nonsingular if the base locus of $L$ does not contain a singular point of $C$, i.e. if for all $x \in C_{\text {sing }}$, there exists an $s \in H^{0}(L)$ such that $s(x) \neq 0$. Finally, $C$ is hyperelliptic if $p_{a}(C) \geq 2$, $C$ is stable, and there is a finite degree two morphism $C \rightarrow \mathbb{P}^{1}$.

Remark 3.3. (i) Suppose that $C$ is stable and that $L$ is a nonsingular $g_{2}^{1}$ on $C$. If $L$ has a base point $x$, necessarily a smooth point of $C$, then $L \otimes \mathcal{O}_{C}(-x)$ has degree one and $h^{0}=2$, and thus defines a morphism $C \rightarrow \mathbb{P}^{1}$ of degree one which is an isomorphism on one component and maps all others to points. Thus, every positive dimensional fiber defines a decomposition $C=C_{1} \cup C_{2}$ with $\#\left(C_{1} \cap C_{2}\right)=1$. In particular, $C$ has a separating node $p$, i.e. a point $p \in C_{\text {sing }}$ such that $C-\{x\}$ is disconnected. (ii) Suppose that $C$ is stable and that $L$ is a base point free $g_{2}^{1}$ on $C$. If the corresponding morphism $C \rightarrow \mathbb{P}^{1}$ is not finite, and $C_{1}$ is a one-dimensional connected component of a fiber, then $C=C_{1} \cup C_{2}$ with $\#\left(C_{1} \cap C_{2}\right) \leq 2$.

We will need the following version of Clifford's theorem for singular curves:

Proposition 3.4. Let $C$ be a nodal curve and $L$ a line bundle on $C$ of degree d. Suppose that, for each irreducible component $C_{i}$ of $C$, there exist sections of $L$ and also of $L^{-1} \otimes \omega_{C}$ which do not vanish identically on $C_{i}$, and hence $0 \leq \operatorname{deg} L\left|C_{i} \leq \operatorname{deg} \omega_{C}\right| C_{i}$ for every $i$. Then

$$
h^{0}(C ; L) \leq \frac{d}{2}+1
$$

Moreover, if $C$ does not have a separating node, then equality holds only if $L=\mathcal{O}_{C}, L=\omega_{C}$, or $C$ has a nonsingular $g_{2}^{1}$. 
Proof. Note that $L$ satisfies the hypotheses of the proposition if and only if $L^{-1} \otimes \omega_{C}$ does, and since $\operatorname{deg}\left(L^{-1} \otimes \omega_{C}\right)=2 p_{a}(C)-2-d$ and $h^{0}(L)=$ $d-p_{a}(C)+1+h^{0}\left(L^{-1} \otimes \omega_{C}\right), L$ satisfies the conclusions of the theorem if and only if $L^{-1} \otimes \omega_{C}$ does.

The proof is by induction on the number of nodes of $C$. The case where there are no nodes is the usual form of Clifford's theorem for a special line bundle $L$. In the general case, if both $L$ and $L^{-1} \otimes \omega_{C}$ are nonsingular, then the result is proved by Beauville [1, Lemma 4.7]. Suppose that either $L$ or $L^{-1} \otimes \omega_{C}$ fails to be nonsingular. By the symmetry between $L$ and $L^{-1} \otimes \omega_{C}$, we may suppose that $L$ is singular.

Let $x \in C_{\text {sing }}$ be such that every section of $H^{0}(L)$ vanishes at $x$. Let $\nu: \hat{C} \rightarrow C$ be the result of normalizing $C$ at $x$, with $\nu^{-1}(x)=\{p, q\}$. By assumption, $\nu^{*} H^{0}(L) \subseteq H^{0}\left(\hat{C} ; \nu^{*} L(-p-q)\right)$, and in fact $\nu^{*} H^{0}(L)=$ $H^{0}\left(\hat{C} ; \nu^{*} L(-p-q)\right)$, so that, for every component $D$ of $\hat{C}$, there exists a section of $\nu^{*} L(-p-q)$ which does not vanish identically on $D$. Set $\hat{L}=\nu^{*} L(-p-q)$. Since $\omega_{\hat{C}}=\nu^{*} \omega_{C}(-p-q),(\hat{L})^{-1} \otimes \omega_{\hat{C}}=\nu^{*}\left(L^{-1} \otimes \omega_{C}\right)$. Thus a section of $L^{-1} \otimes \omega_{C}$ which does not vanish on any component of $C$ defines a section of $(\hat{L})^{-1} \otimes \omega_{\hat{C}}$ with the same property, so that $\hat{L}$ satisfies the inductive hypothesis.

Case 1: $\hat{C}$ is connected, i.e. $x$ is not a separating node. Then by the inductive hypothesis,

$$
h^{0}(L)=h^{0}(\hat{C} ; \hat{L}) \leq \frac{d-2}{2}+1=\frac{d}{2}<\frac{d}{2}+1 .
$$

Hence strict inequality holds in this case.

Case 2: $\hat{C}$ is not connected, i.e. $x$ is a separating node. Let $C_{1}, C_{2}$ be the connected components of $\hat{C}$. Let $L_{i}=\hat{L} \mid C_{i}$ and let $d_{i}=\operatorname{deg}\left(L \mid C_{i}\right)$. Then $\operatorname{deg} L_{i}=d_{i}-1$ and $d=d_{1}+d_{2}$. Again, $\hat{L}$ satisfies the hypotheses of Clifford's theorem, so that, by the inductive hypothesis,

$$
h^{0}(L)=h^{0}\left(C_{1} ; L_{1}\right)+h^{0}\left(C_{2} ; L_{2}\right) \leq \frac{d_{1}-1}{2}+1+\frac{d_{2}-1}{2}+1=\frac{d}{2}+1 .
$$

Thus the conclusion holds in this case as well. Moreover, if there is no separating node, then the proof of Case I shows that equality holds only in the nonsingular case, and then by Beauville's result either $L$ is $\mathcal{O}_{C}$ or $\omega_{C}$ or $C$ has a nonsingular $g_{2}^{1}$.

Lemma 3.5. Let $\pi: \widetilde{C} \rightarrow C$ be an admissible double cover satisfying condition $(*)$ of Beauville. If $\widetilde{C}$ has a nonsingular $g_{2}^{1}$, then $C$ has a nonsingular $g_{2}^{1}$. 
Proof. We shall just write out the proof in the case where the nonsingular $g_{2}^{1}$ defines a finite morphism $f: \widetilde{C} \rightarrow \mathbb{P}^{1}$, the only case we shall need. In this case, if $p$ and $q$ are smooth points of $\widetilde{C}$ such that $f(p)=f(q)$, then in the usual way $h^{0}\left(\omega_{\widetilde{C}}(-p-q)\right)=h^{0}\left(\omega_{\widetilde{C}}\right)-1$, i.e. a section of $\omega_{\widetilde{C}}$ which vanishes at $p$ also vanishes at $q$. If $\pi(p) \neq \pi(q)$, since $\pi^{*} \omega_{C}=\omega_{\widetilde{C}}$, it follows that every section of $\omega_{C}$ vanishing at $\pi(p)$ vanishes at $\pi(q)$ and $C$ is hyperelliptic. Otherwise, for every choice of smooth points $p$ and $q$ as above, if $f(p)=f(q)$, then $\pi(p)=\pi(q)$. But then $f$ factors through $C$ and induces a finite, degree one morphism from $C$ to $\mathbb{P}^{1}$, which is impossible.

\subsection{Invariant line bundles}

We begin with the following situation: $\pi: \hat{C} \rightarrow N$ is a smooth connected double cover, corresponding to the involution $\tau$ on $\hat{C}$. Suppose that the fixed points of $\tau$ are $p_{1}, \ldots, p_{2 k}$ where $k>0$. Let $L$ be a line bundle on $\hat{C}$ such that $\tau^{*} L=L$ in Pic $\hat{C}$, and let $(\operatorname{Pic} \hat{C})^{\tau}$ be the subgroup of all invariant line bundles. We define an invariant $\alpha(L)$ as follows: choose an isomorphism $\varphi: \tau^{*} L \rightarrow L$ such that $\varphi^{2}=\mathrm{Id}$, where $\varphi^{2}$ is understood to be the composition

$$
L=\left(\tau^{*}\right)^{2} L \stackrel{\tau^{*} \varphi}{\longrightarrow} \tau^{*} L \stackrel{\varphi}{\rightarrow} L .
$$

Such a $\varphi$ is unique up to \pm 1 . For a fixed point $p_{i}, \varphi_{p_{i}}: L_{p_{i}} \rightarrow L_{p_{i}}$ is multiplication by \pm 1 . Thus

$$
\alpha(L)=\left(\varphi_{p_{1}}, \ldots, \varphi_{p_{2 k}}\right) \in(\{ \pm 1\})^{2 k} /( \pm \mathrm{Id})
$$

is well-defined, where $\operatorname{Id}=(1, \ldots, 1)$ and similarly for $-\mathrm{Id}$. Define the homomorphism $d:(\{ \pm 1\})^{2 k} \rightarrow\{ \pm 1\}$ by $d\left(\epsilon_{1}, \ldots, \epsilon_{2 k}\right)=\epsilon_{1} \cdots \epsilon_{2 k}$. Note that $d$ factors through the quotient $(\{ \pm 1\})^{2 k} /( \pm \mathrm{Id})$. The induced homomorphism $(\{ \pm 1\})^{2 k} /( \pm \mathrm{Id}) \rightarrow\{ \pm 1\}$ will again be denoted by $d$.

Proposition 3.6. With notation as above, $\alpha$ : $(\operatorname{Pic} \hat{C})^{\tau} \rightarrow(\{ \pm 1\})^{2 k} /( \pm \mathrm{Id})$ is a surjective homomorphism satisfying

$$
d(\alpha(L))=(-1)^{\operatorname{deg}(L)} .
$$

If $1 \leq i_{1}<\cdots<i_{\ell} \leq 2 k$, then $\alpha\left(\mathcal{O}_{\hat{C}}\left(p_{i_{1}}+\cdots+p_{i_{\ell}}\right)\right)$ is represented by $\left(\epsilon_{1}, \ldots, \epsilon_{2 k}\right)$, where $\epsilon_{i}=-1$ if and only if $i=i_{j}$ for some $j$. Finally, $\operatorname{Ker} \alpha=\pi^{*} \operatorname{Pic} N$.

Proof. It is easy to check that $\alpha$ is a homomorphism. The natural linearization $\varphi$ on $\mathcal{O}_{\hat{C}}\left(p_{i_{1}}+\cdots+p_{i_{\ell}}\right)$ induced by $f \mapsto \tau^{*} f$, where $f$ is a meromorphic 
function on a Zariski open subset of $\hat{C}$ with at worst simple poles along the $p_{i_{j}}$, has the property that it induces -1 on the fibers over the $p_{i_{j}}$, since there exists an anti-invariant local uniformizing parameter over such points, and is 1 over the remaining fixed points. In particular, $\alpha$ is surjective and $d\left(\alpha\left(\mathcal{O}_{\hat{C}}\left(p_{i_{1}}+\cdots+p_{i_{\ell}}\right)\right)=(-1)^{\ell}\right.$.

The line bundle $L \in \operatorname{Ker} \alpha$ if and only if there is a linearization $\varphi$ which is the identity over the fixed points. By descent theory, this condition is equivalent to $L=\pi^{*} M$ for some $M \in \operatorname{Pic} N$. Thus the set of line bundles $\left\{\mathcal{O}_{\hat{C}}\left(p_{i_{1}}+\cdots+p_{i_{\ell}}\right)\right\}$ contains a set of representatives for $(\operatorname{Pic} \hat{C})^{\tau} / \pi^{*} \operatorname{Pic} N$, and these representatives are unique modulo the relation $\mathcal{O}_{\hat{C}}\left(p_{1}+\cdots+p_{2 k}\right)=$ $\pi^{*} \mathcal{O}_{N}(B)$ for the divisor class $B$ which defines the double cover $\hat{C}$, i.e. $\mathcal{O}_{N}(2 B) \cong \mathcal{O}_{N}\left(q_{1}+\cdots+q_{2 k}\right)$ where $q_{i}=\pi\left(p_{i}\right)$. The formula $d(\alpha(L))=$ $(-1)^{\operatorname{deg}(L)}$ then holds for all $L$, since it holds for $L=\pi^{*} M \in \pi^{*} \operatorname{Pic} N$ since $\operatorname{deg} \pi^{*} M$ is even, and it also holds for a set of coset representatives of $(\operatorname{Pic} \hat{C})^{\tau} / \pi^{*} \operatorname{Pic} N$.

We will also need to look at orders of vanishing of invariant or antiinvariant sections.

Lemma 3.7. Let $\varphi$ be a linearization on $L$ and let $p$ be a fixed point of $\tau$.

(i) If $\varphi_{p}=1$, then every anti-invariant section $s$ of $L$ vanishes at $p$, and every invariant section $s$ of $L$ which vanishes at $p$ vanishes to order at least two at $p$.

(ii) If $\varphi_{p}=-1$, then every invariant section $s$ of $L$ vanishes at $p$, and every anti-invariant section $s$ of $L$ which vanishes at $p$ vanishes to order at least two at $p$.

Proof. First suppose that $\varphi_{p}=1$. Let $\sigma_{0}$ be a local generating section of $L$ at $p$. By replacing $\sigma_{0}$ with $\sigma_{0}+\varphi\left(\tau^{*} \sigma_{0}\right)$ and shrinking further to a $\tau$-invariant open set $U$ containing $p$, we may assume that $\varphi\left(\tau^{*} \sigma\right)=\sigma$. If $s=f \sigma$ for some holomorphic function $f$ on $U$, then $s$ is invariant if and only if $\tau^{*} f=f$ and $s$ is anti-invariant if and only if $\tau^{*} f=-f$. In the second case we see that $f$ vanishes at $p$, and in the first case, if $f$ vanishes at $p$, then it vanishes to order at least 2 there.

The case where $\varphi_{p}=-1$ follows from the case $\varphi_{p}=1$ by replacing $\varphi$ by $-\varphi$.

We now consider the case where $\pi: \widetilde{C} \rightarrow C$ is an admissible double cover of the nodal curve $C$ satisfying $(*)$ and corresponding to the involution $\tau$. Let $\nu: \hat{C} \rightarrow \widetilde{C}$ be the (possibly disconnected) normalization of $\widetilde{C}$, so 
that there is also the induced double cover $\hat{C} \rightarrow N$ and the corresponding involution, which we continue to denote by $\pi$ and $\tau$. It is not always the case that a $\tau$-invariant line bundle $\hat{L}$ on $\hat{C}$ lifts to a $\tau$-invariant line bundle on $\widetilde{C}$. The condition is as follows:

Proposition 3.8. Let $\hat{L} \in(\operatorname{Pic} \hat{C})^{\tau}$. Then $\hat{L}$ lifts to a $\tau$-invariant line bundle on $\widetilde{C}$ if and only if there exists a linearization $\hat{\varphi}$ of $\hat{L}$ such that, for every double point $x$ of $\widetilde{C}$, if $\nu^{-1}(x)=\{p, q\}$, then $\hat{\varphi}_{p}=\hat{\varphi}_{q}$. Hence, if $L$ is a $\tau$-invariant line bundle on $\widetilde{C}$, then the pullback of $L$ to $\hat{C}$ is of the form $\pi^{*} \hat{M} \otimes \mathcal{O}_{\hat{C}}\left(\sum_{i=1}^{k}\left(p_{i}+q_{i}\right)\right)$, where $x_{1}, \ldots, x_{k}$ are distinct nodes of $\widetilde{C}$ and $\nu^{-1}\left(x_{i}\right)=\left\{p_{i}, q_{i}\right\}$.

Proof. If $\tau^{*} L \cong L$, then we can choose a linearization $\varphi$ on $L$ which then pulls back to a linearization $\hat{\varphi}$ of $\hat{L}$ satisfying $\hat{\varphi}_{p}=\hat{\varphi}_{q}$ for every pair of points $p, q \in \hat{C}$ which are identified under $\nu$. Conversely, such a linearization $\hat{\varphi}$ clearly induces an isomorphism from $\tau^{*} L$ to $L$.

Remark 3.9. It is easy to see that, if one lift of $\hat{L}$ is $\tau$-invariant, then all lifts are $\tau$-invariant.

Next, we need to know how to compute $\pi_{*} L$ for a $\tau$-invariant $L$.

Proposition 3.10. Suppose that $\widetilde{C}_{\text {sing }}=\left\{x_{1}, \ldots, x_{n}\right\}$ and set $\nu^{-1}\left(x_{i}\right)=$ $\left\{p_{i}, q_{i}\right\}$. Let $L$ be a $\tau$-invariant line bundle such that $\nu^{*} L \cong \pi^{*} \hat{M}_{1}\left(p_{1}+q_{1}+\right.$ $\left.\cdots+p_{k}+q_{k}\right)$. Thus we can also write $\nu^{*} L \cong \pi^{*} \hat{M}_{2}\left(p_{k+1}+q_{k+1}+\cdots+p_{n}+q_{n}\right)$. Let $\nu_{1}: N_{1} \rightarrow C$ be the normalization of $C$ at $\pi\left(x_{1}\right), \ldots, \pi\left(x_{k}\right)$ and let $M_{1}$ be the line bundle on $N_{1}$ obtained from $\hat{M}_{1}$ by using the identifications $L_{p_{k+1}} \cong$ $L_{q_{k+1}}, \ldots, L_{p_{n}} \cong L_{q_{n}}$. Let $\nu_{2}: N_{2} \rightarrow C$ be the normalization of $C$ at $\pi\left(x_{k+1}\right), \ldots, \pi\left(x_{n}\right)$ and let $M_{2}$ be the line bundle on $N_{2}$ obtained from $\hat{M}_{2}$ by using the identifications $L_{p_{1}} \cong L_{q_{1}}, \ldots, L_{p_{k}} \cong L_{q_{k}}$. Then

$$
\pi_{*} L \cong \nu_{1 *} M_{1} \oplus \nu_{2 *} M_{2}
$$

where, possibly after replacing $\varphi$ by $-\varphi, \nu_{1 *} M_{1}$ is the +1 -eigenspace for the action of $\varphi$ and $\nu_{2 *} M_{2}$ is the-1-eigenspace. Finally, if Norm $L=\omega_{C}$, then $M_{1}$ is a theta characteristic on $N_{1}$ and $M_{2}$ is a theta characteristic on $N_{2}$.

Proof. This is essentially a local computation at each singular point. Let $R=\mathbb{C}[[x, y]] /(x y)$ and let $S=\mathbb{C}[[u, v]] /(u v)$, and view $R$ as included in $S$ via $x=u^{2}, y=v^{2}$. There is a natural involution $\tau$ on $S$, defined by $\tau(u)=-u$ and $\tau(v)=-v$, and $R$ is the ring of $\tau$-invariants. 
First consider $S$ as a module over $R$. Then $S=R \oplus(u \mathbb{C}[[x]] \oplus v \mathbb{C}[[y]])$. In invariant terms, this says that

$$
\pi_{*} \mathcal{O}_{\widetilde{C}}=\mathcal{O}_{C} \oplus \mathcal{O}_{N}(-B),
$$

where $B$ is the line bundle on $N$ which is the square root of the branch divisor on $\hat{C} \rightarrow N$. Moreover, the first factor is the +1 -eigenspace and the second is the -1-eigenspace. More generally, by the projection formula,

$$
\pi_{*} \pi^{*} M=M \oplus\left(M \otimes \mathcal{O}_{N}(-B)\right),
$$

where in the usual linearization the first factor is the +1-eigenspace and the second is the -1-eigenspace.

Now suppose that $L$ is a line bundle on $C$ such that $\hat{L} \cong \mathcal{O}_{\hat{C}}(p+q)$. Locally, there exists a $c \in \mathbb{C}^{*}$ such that $L$ corresponds to the $S$-submodule $X$ of $\mathbb{C}[[u]] \cdot \frac{1}{u} \oplus \mathbb{C}[[v]] \cdot \frac{1}{v}$ consisting of all $\left(f_{1}, f_{2}\right)$ such that, if the leading term of $f_{1}$ is $a / u$, then that of $f_{2}$ is $c a / v$, i.e.

$$
X=\left\{a \alpha+g_{1}+g_{2}: g_{1} \in \mathbb{C}[[u]], g_{2} \in \mathbb{C}[[v]], a \in \mathbb{C}\right\},
$$

where $\alpha=1 / u+c / v$, so that $\tau(\alpha)=-\alpha$. As an $R$-module,

$$
X=(\mathbb{C}[[x]] \oplus \mathbb{C}[[y]]) \oplus(\mathbb{C} \cdot \alpha \oplus u \cdot \mathbb{C}[[x]] \oplus v \mathbb{C}[[y]])=X_{+} \oplus X_{-},
$$

say, where $X_{+}$is $\tau$-invariant and $X_{-}$is $\tau$-anti-invariant. More intrinsically, $X_{+} \cong \widetilde{R}$, the normalization of $R$, and $X_{-} \cong R$. These two cases together say that, if $L$ is a line bundle such that $\nu^{*} L=\mathcal{O}_{\hat{C}}\left(p_{1}+q_{1}+\cdot+p_{k}+q_{k}\right)$ with the natural linearization, then the +1 -eigenspace of $\pi_{*} L$ is isomorphic to $\nu_{1 *} \mathcal{O}_{N_{1}}$. Likewise, if $\nu^{*} L=\left(\pi^{*} \hat{M}_{1}\right) \otimes \mathcal{O}_{\hat{C}}\left(p_{1}+q_{1}+\cdot+p_{k}+q_{k}\right)$ with the natural linearization, then the +1 -eigenspace of $\pi_{*} L$ is isomorphic to $\nu_{1 *} M_{1}$. By symmetry, a similar statement holds for the -1-eigenspace.

To see the final statement, let $\hat{L}_{1}$ be the pullback of $L$ to the normalization $N_{1}$ of $C$ at $x_{1}, \ldots, x_{k}$, and similarly for $\hat{L}_{2}$. Then since Norm $L=\omega_{C}$,

$$
\text { Norm } \hat{L}_{1}=\nu_{1}^{*} \omega_{C}=\omega_{N_{1}}\left(\pi\left(p_{1}\right)+\pi\left(q_{1}\right)+\cdots+\pi\left(p_{k}\right)+\pi\left(q_{k}\right)\right) .
$$

But we also have $\hat{L}_{1}=\pi^{*} M_{1}\left(p_{1}+q_{1}+\cdots+p_{k}+q_{k}\right)$, so that

$$
\text { Norm } \hat{L}_{1}=M_{1}^{\otimes 2}\left(\pi\left(p_{1}\right)+\pi\left(q_{1}\right)+\cdots+\pi\left(p_{k}\right)+\pi\left(q_{k}\right)\right) \text {. }
$$

Comparing, we see that $M_{1}^{\otimes 2}=\omega_{N_{1}}$. A similar statement works for $M_{2}$.

We will also use the following: 
Lemma 3.11. Let $L, N_{i}$ and $M_{i}$ be as in the previous lemma. Suppose that, for every irreducible component $D$ of $C, \operatorname{deg}(L \mid \widetilde{D})$ is even, where $\widetilde{D}$ is the component of $\widetilde{C}$ corresponding to $D$. Suppose further that $x_{1}, \ldots, x_{\ell}$ are the points lying on $D$ where $\nu_{1}: N_{1} \rightarrow C$ is not an isomorphism. Then:

(i) $\ell=2 k$ is even, and $\operatorname{deg}\left(M_{1} \mid D\right)=\frac{1}{2} \operatorname{deg}(L \mid \widetilde{D})-k$.

(ii) If $N_{i}=N^{\prime} \cup N^{\prime \prime}$, where both $N^{\prime}$ and $N^{\prime \prime}$ are a union of components of $N_{i}$ and $N^{\prime} \cap N^{\prime \prime}$ is finite, then $\#\left(N^{\prime} \cap N^{\prime \prime}\right)$ is even.

(iii) Every connected component of $N_{i}$ is either smooth rational or semistable with arithmetic genus at least one.

Proof. (i) follows from the definitions and the fact that $d(\alpha(L))=1$. (ii) is clear if, say, $N^{\prime}$ is irreducible, and follows in the general case by a straightforward induction on the number of components of $N^{\prime}$. (iii) is then clear.

\subsection{Genus six}

Our goal is now to prove:

Theorem 3.12. Let $\pi: \widetilde{C} \rightarrow C$ be an admissible double cover of a stable curve $C$ with $p_{a}(C)=6$. If the theta divisor $\Xi$ of the associated Prym variety $P(\widetilde{C}, \pi)$ has a unique singular point which is a triple point, then $C$ is a plane quintic and $\pi: \widetilde{C} \rightarrow C$ is the double cover associated to an odd theta characteristic.

To prove Theorem 3.12 we may assume that $\pi$ satisfies condition $(*)$ and that, for every decomposition $\widetilde{C}=\widetilde{C}_{1} \cup \widetilde{C}_{2}$, with $\widetilde{C}_{1} \cap \widetilde{C}_{2}$ finite, then $\#\left(\widetilde{C}_{1} \cap \widetilde{C}_{2}\right) \geq 4$. Let $\Xi_{\text {sing }}=\{x\}$, so that $\tau(x)=x$. Let $L \in \operatorname{Pic} \widetilde{C}$ be the corresponding line bundle, so that $\operatorname{deg} L=10, h^{0}(L)=2 n$ is even, Norm $L=\omega_{C}$ and $\tau^{*} L=L$. Thus $L=\omega_{\widetilde{C}} \otimes L^{-1}$. Via the isomorphism $H^{0}(L)=H^{0}\left(M_{1}\right) \oplus H^{0}\left(M_{2}\right)$ of Proposition 3.10, we can write

$$
\operatorname{dim} H^{0}(L)=2 n=\operatorname{dim} H^{0}\left(M_{1}\right)+\operatorname{dim} H^{0}\left(M_{2}\right)=n_{1}+n_{2},
$$

say, where $n_{i}=\operatorname{dim} H^{0}\left(M_{i}\right)$. Possibly after relabeling, we may assume that $n_{1} \geq n_{2}$. Then Theorem 3.12 follows from:

Theorem 3.13. With the above notation,

(i) $2 n=h^{0}(L) \leq 6$, and equality holds only if $C$ is hyperelliptic. In this case $\operatorname{dim} \Xi_{\text {sing }} \geq 1$. 
(ii) Suppose that $h^{0}(L)=4$.

(a) The case $\left(n_{1}, n_{2}\right)=(4,0)$ does not arise.

(b) If $\left(n_{1}, n_{2}\right)=(3,1)$, then either $C$ is a plane quintic and $L=\pi^{*} M$, where $M$ is the unique line bundle of degree 5 with $h^{0}(M)=3$ on $C$, or $\operatorname{dim} \Xi_{\text {sing }} \geq 1$.

(c) If $\left(n_{1}, n_{2}\right)=(2,2)$, then $L$ corresponds to a point of multiplicity 2 of $\Xi$.

(iii) Suppose that $h^{0}(L)=2$.

(a) If $\left(n_{1}, n_{2}\right)=(2,0)$, then $L$ corresponds to a point of multiplicity 2 of $\Xi$.

(b) If $\left(n_{1}, n_{2}\right)=(1,1)$, then $L$ corresponds to a smooth point of $\Xi$.

We now begin the proof of Theorem 3.13

Lemma 3.14. Let $s$ be a nonzero element of $H^{0}(L)$, and suppose that $\widetilde{C}=$ $C_{s}^{\prime} \cup C_{s}^{\prime \prime}$, where $C_{s}^{\prime}$ is the union of the irreducible components of $\widetilde{C}$ where $s$ vanishes identically, $C_{s}^{\prime \prime}$ is the union of the irreducible components of $\widetilde{C}$ where the restriction of $s$ is not zero, and hence $C_{s}^{\prime} \cap C_{s}^{\prime \prime}$ is finite. Then:

(i) $C_{s}^{\prime \prime}$ is connected.

(ii) Let $C_{s}^{\prime} \cap C_{s}^{\prime \prime}=\left\{x_{1}, \ldots, x_{b}\right\}$ and let $L^{\prime \prime}=\left(L \mid C_{s}^{\prime \prime}\right)\left(-\sum_{i} x_{i}\right)$. Then $s$ defines sections of $L^{\prime \prime}$ and $\left(L^{\prime \prime}\right)^{-1} \otimes \omega_{C_{s}^{\prime \prime}}$ which do not vanish on any component of $C_{s}^{\prime \prime}$.

Proof. It suffices to consider the case when $C_{s}^{\prime}$ is not empty. If $D$ is a connected component of $C_{s}^{\prime \prime}$, then the intersection of $D$ with the remaining components of $\widetilde{C}$ is the same as the intersection of $D$ with $C_{s}^{\prime}$, and thus $\#\left(D \cap C_{s}^{\prime}\right) \geq 4$. Hence $\operatorname{deg}(L \mid D) \geq 4$. By Lemma 3.1. $L$ has degree at least two on every irreducible component of $C_{s}^{\prime}$. Thus, if $C_{s}^{\prime \prime}$ has at least two connected components, the only possibility is that $C_{s}^{\prime \prime}$ has exactly two connected components $D_{1}$ and $D_{2}, L \mid D_{i}$ has degree 4 for $i=1,2, C_{s}^{\prime}$ is irreducible, $\#\left(C_{s}^{\prime} \cap C_{s}^{\prime \prime}\right)=8$, and $\operatorname{deg}\left(L \mid C_{s}^{\prime}\right)=2$. But by Lemma 3.1] $\operatorname{deg}\left(L \mid C_{s}^{\prime}\right)=2=2 \bar{p}-2+\#\left(C_{s}^{\prime} \cap C_{s}^{\prime \prime}\right)$, where $\bar{p}$ is the arithmetic genus of the image of $C_{s}^{\prime}$ in $C$. Thus $\#\left(C_{s}^{\prime} \cap C_{s}^{\prime \prime}\right)=4-2 \bar{p} \leq 4$, contradicting $\#\left(C_{s}^{\prime} \cap C_{s}^{\prime \prime}\right)=8$. Hence $C_{s}^{\prime \prime}$ is connected, proving (i). 
Clearly the section $s$ does not vanish on any component of $C_{s}^{\prime \prime}$. Since

$$
\begin{aligned}
\left(L \mid C_{s}^{\prime \prime}\right)^{-1} \otimes \omega_{C_{s}^{\prime \prime}} & =\left(L \mid C_{s}^{\prime \prime}\right)^{-1} \otimes\left(\omega_{\widetilde{C}} \mid C_{s}^{\prime \prime}\right)\left(-\sum_{i} x_{i}\right) \\
& =\left(L^{-1} \otimes \omega_{\widetilde{C}} \mid C_{s}^{\prime \prime}\right)\left(-\sum_{i} x_{i}\right)=\left(L \mid C_{s}^{\prime \prime}\right)\left(-\sum_{i} x_{i}\right)=L^{\prime \prime},
\end{aligned}
$$

it follows that $\left(L^{\prime \prime}\right)^{-1} \otimes \omega_{C_{s}^{\prime \prime}}=L^{\prime \prime}\left(\sum_{i} x_{i}\right)$. The section $s$ then defines a section of $L^{\prime \prime}\left(\sum_{i} x_{i}\right)=\left(L^{\prime \prime}\right)^{-1} \otimes \omega_{C_{s}^{\prime \prime}}$ which does not vanish on any component.

Corollary 3.15. If there exists a component of $\widetilde{C}$ on which every section $s$ of $L$ vanishes, then $h^{0}(L) \leq 2$.

Proof. As in the previous lemma, write $\widetilde{C}=C^{\prime} \cup C^{\prime \prime}$, where $C^{\prime}$ is the union of all components of $\widetilde{C}$ where all sections of $L$ vanish, or equivalently where a generic section of $L$ vanishes, and let $C^{\prime} \cap C^{\prime \prime}=\left\{x_{1}, \ldots, x_{b}\right\}$. Then $\left(L \mid C^{\prime \prime}\right)\left(-\sum_{i} x_{i}\right)$ has degree at most $8-4=4$ and satisfies the hypotheses of Proposition 3.4. Thus $h^{0}(L)=h^{0}\left(\left(L \mid C^{\prime \prime}\right)\left(-\sum_{i} x_{i}\right)\right) \leq 3$, and so $h^{0}(L) \leq$ 2 .

Corollary 3.16. We have $h^{0}(L) \leq 6$, and equality holds only if $\widetilde{C}$ and hence $C$ are hyperelliptic.

Proof. This is immediate from the previous corollary, Remark 3.3. Proposition 3.4 and Lemma 3.5 .

Proposition 3.17. Suppose that $s_{+} \in H^{0}(L)$ is a nonzero invariant section and that $s_{-} \in H^{0}(L)$ is a nonzero anti-invariant section. Then there exists a component $D$ of $\widetilde{C}$ such that neither $s_{+}$nor $s_{-}$restricts to 0 on $D$.

Proof. Suppose to the contrary that, for every component $D$ of $\widetilde{C}$ such that $s_{+}$restricts non-trivially to $D, s_{-}$restricts to 0 on $D$ and vice-versa. Let $C_{s_{+}}^{\prime \prime}$ be the union of the components of $\widetilde{C}$ where $s_{+}$restricts non-trivially, and similarly for $C_{s_{-}}^{\prime \prime}$. By assumption, $C_{s_{+}}^{\prime \prime}$ and $C_{s_{-}}^{\prime \prime}$ meet in a finite number (possibly zero) of points, and by Lemma [3.14, $C_{s_{+}}^{\prime \prime}$ and $C_{s_{-}}^{\prime \prime}$ are connected. Let $s$ be a generic linear combination of $s_{+}$and $s_{-}$, and let $C_{s}^{\prime \prime}$ be the union of the components of $\widetilde{C}$ where $s$ has non-trivial restriction. Clearly $C_{s}^{\prime \prime}=C_{s_{+}}^{\prime \prime} \cup C_{s_{-}}^{\prime \prime}$. Again by Lemma 3.14. $C_{s}^{\prime \prime}$ is connected. It follows that $C_{s_{+}}^{\prime \prime} \cap C_{s_{-}}^{\prime \prime} \neq \emptyset$.

Let $C_{s}^{\prime}$ be the union of the components where $s$ restricts to 0. Clearly, $C_{s}^{\prime}$ is the intersection of the corresponding subschemes $C_{s_{+}}^{\prime}$ and $C_{s_{-}}^{\prime}$, and $C=C_{s}^{\prime} \cup C_{s_{+}}^{\prime \prime} \cup C_{s_{-}}^{\prime \prime}$, where the pairwise intersections of $C_{s}^{\prime}, C_{s_{+}}^{\prime \prime}$, and $C_{s_{-}}^{\prime \prime}$ are 
finite. First assume that $C_{s}^{\prime} \neq \emptyset$. Then as $\operatorname{deg}\left(L \mid C_{s_{+}}^{\prime \prime}\right) \geq 4 \operatorname{deg}\left(L \mid C_{s_{-}}^{\prime \prime}\right) \geq 4$, and $\operatorname{deg}\left(L \mid C_{s}^{\prime}\right) \geq 2$, we must have $\operatorname{deg}\left(L \mid C_{s_{+}}^{\prime \prime}\right)=\operatorname{deg}\left(L \mid C_{s_{-}}^{\prime \prime}\right)=4$, and both $C_{s_{+}}^{\prime \prime}$ and $C_{s_{-}}^{\prime \prime}$ meet the remaining components of $\widetilde{C}$ in 4 points, at least one of which lies on $C_{s_{+}}^{\prime \prime} \cap C_{s_{-}}^{\prime \prime}$. Let $C_{s_{+}}^{\prime \prime} \cap \overline{\left(\widetilde{C}-C_{s_{+}}^{\prime \prime}\right)}=\left\{x_{1}, x_{2}, x_{3}, p\right\}$, say, where $p$ corresponds to a point of $C_{s_{+}}^{\prime \prime} \cap C_{s_{-}}^{\prime \prime}$, and let $C_{s_{-}}^{\prime \prime} \cap \overline{\left(\widetilde{C}-C_{s_{-}}^{\prime \prime}\right)}=\left\{y_{1}, y_{2}, y_{3}, q\right\}$, say, where $q$ corresponds to the point $p$. Let $\varphi$ be a choice of linearization for $L$ and let $\hat{\varphi}_{ \pm}$be the corresponding linearizations on $C_{s_{ \pm}}^{\prime \prime}$. By Proposition 3.8 $\left(\hat{\varphi}_{+}\right)_{p}=\left(\hat{\varphi}_{-}\right)_{q}$. It then follows from Lemma 3.7 that either $s_{+}$vanishes to order at least two at $p$ or $s_{-}$vanishes to order at least two at $q$. In particular, either $\operatorname{deg}\left(L \mid C_{s_{+}}^{\prime \prime}\right) \geq 5$ or $\operatorname{deg}\left(L \mid C_{s_{-}}^{\prime \prime}\right) \geq 5$. This is a contradiction, and hence there exists a component $D$ of $\widetilde{C}$ where both $s_{+}$and $s_{-}$restrict non-trivially.

Finally assume that $C_{s}^{\prime}=\emptyset$. Then $\widetilde{C}=C_{s_{+}}^{\prime \prime} \cup C_{s_{-}}^{\prime \prime}$, and $\#\left(C_{s_{+}}^{\prime \prime} \cap C_{s_{-}}^{\prime \prime}\right) \geq 4$. At each point $p$ of $C_{s_{+}}^{\prime \prime} \cap C_{s_{-}}^{\prime \prime}$, viewed as a point of $C_{s_{+}}^{\prime \prime}, s_{+}$vanishes, and a similar statement holds at the corresponding point $q$ of $C_{s_{-}}^{\prime \prime}$. Moreover, again by Lemma 3.7 either $s_{+}$vanishes to order at least two at $p$ or $s_{-}$vanishes to order at least two at $q$. It follows that $\operatorname{deg}\left(L \mid C_{s_{+}}^{\prime \prime}\right)+\operatorname{deg}\left(L \mid C_{s_{-}}^{\prime \prime}\right) \geq 4(3)=12$, which contradicts $\operatorname{deg} L=10$. Thus, again there exists a component $D$ of $\widetilde{C}$ where both $s_{+}$and $s_{-}$restrict non-trivially.

Completion of the proof of Theorem 3.13. We have already dealt with the case $h^{0}(L) \geq 6$. Next suppose that $h^{0}(L)=2$ and $\left(n_{1}, n_{2}\right)=(2,0)$. In this case, with notation as in Proposition 3.10 given $q \in C$ a smooth point such that $\pi^{-1}(q)=\{p, \tau(p)\}, H^{0}\left(L \otimes \mathcal{O}_{\widetilde{C}}(2 p+2 \tau(p))\right)^{+}=H^{0}\left(M_{1} \otimes \mathcal{O}_{N_{1}}(2 q)\right)$. Since $h^{0}\left(M_{1}\right)=2$, we may choose $q$ so that $h^{1}\left(M_{1}(2 q)\right)=h^{0}\left(M_{1}(-2 q)\right)=0$. The method of proof of Lemma 2.6] and Theorem 2.4 in the case of a singular curve shows that $L$ corresponds to a point of $\Xi$ of multiplicity two. If $h^{0}(L)=2$ and $\left(n_{1}, n_{2}\right)=(1,1)$, then up to scalars there are unique nonzero sections $s_{+} \in H^{0}(L)^{+}, s_{-} \in H^{0}(L)^{-}$and there exists a component $D$ of $\widetilde{C}$ such that neither $s_{+}$nor $s_{-}$restricts to 0 on $D$. After relabeling, we may assume that $H^{0}(L)^{+}=H^{0}\left(M_{1}\right)$ and that $H^{0}(L)^{-}=H^{0}\left(M_{2}\right)$. For a general point $p \in D$, lying over $q \in C$, we can assume that $H^{0}\left(M_{1}(-q)\right)=$ $H^{0}\left(M_{2}(-q)\right)=0$. Thus

$$
H^{0}(L(-p-\tau(p)))=H^{0}\left(M_{1}(-q)\right) \oplus H^{0}\left(M_{2}(-q)\right)=0,
$$

so that $p$ and $\tau(p)$ impose independent conditions on $H^{0}(L)$. It then follows from Theorem 2.3 that the multiplicity of $\Xi$ at the point corresponding to $L$ is one. (Alternatively, one could use Mumford's tangent cone condition as proved by Beauville [1, (4.1)] in the case of an admissible double cover.) 
Now suppose that $h^{0}(L)=4$. If $\left(n_{1}, n_{2}\right)=(2,2)$, we claim that there exist points $q_{1}, q_{2} \in C$, with preimages $\pi^{-1}\left(q_{i}\right)=\left\{p_{i}, \tau\left(p_{i}\right)\right\}$, such that the points $p_{1}, \tau\left(p_{1}\right), p_{2}, \tau\left(p_{2}\right)$ impose independent conditions on $H^{0}(L)$. Then, again by Theorem 2.3 it follows that the multiplicity of $\Xi$ at the point corresponding to $L$ is two. To find the points $q_{1}, q_{2}$, begin with a component $D_{1}$ of $\widetilde{C}$ such that there exist sections both in $H^{0}(L)^{+}$and $H^{0}(L)^{-}$whose restrictions to $D_{1}$ are not zero, and choose $q_{1}$ to be a general point of the image of $D_{1}$ in $C$. The arguments above for the case $\left(n_{1}, n_{2}\right)=(1,1)$ show that

$$
H^{0}\left(L\left(-p_{1}-\tau\left(p_{1}\right)\right)\right)=H^{0}\left(M_{1}\left(-q_{1}\right)\right) \oplus H^{0}\left(M_{2}\left(-q_{1}\right)\right)
$$

where now $\operatorname{dim} H^{0}\left(M_{i}\left(-q_{1}\right)\right)=1$. Choose a nonzero section

$$
s_{+} \in H^{0}\left(L\left(-p_{1}-\tau\left(p_{1}\right)\right)\right)^{+}=H^{0}\left(M_{1}\left(-q_{1}\right)\right),
$$

and choose $s_{-}$similarly. In particular, we can view $s_{ \pm}$as nonzero sections of $L$. By Proposition 3.17 there exists a component $D_{2}$ (possibly equal to $D_{1}$ ) of $\widetilde{C}$ such that both $s_{+}$and $s_{-}$have nonzero restriction to $D_{2}$. Let $q_{2}$ be a general point of the image of $D_{2}$ in $C$ and let $\pi^{-1}\left(q_{2}\right)=\left\{p_{2}, \tau\left(p_{2}\right)\right\}$. Then

$H^{0}\left(L\left(-p_{1}-\tau\left(p_{1}\right)-p_{2}-\tau\left(p_{2}\right)\right)\right)=H^{0}\left(M_{1}\left(-q_{1}-q_{2}\right)\right) \oplus H^{0}\left(M_{2}\left(-q_{1}-q_{2}\right)\right)=0$.

Hence $p_{1}, \tau\left(p_{1}\right), p_{2}, \tau\left(p_{2}\right)$ impose independent conditions on $H^{0}(L)$, so that $L$ corresponds to a point of multiplicity two.

Thus we may henceforth assume that $\left(n_{1}, n_{2}\right)=(3,1)$ or $(4,0)$. It will be convenient to divide the rest of the argument into cases.

Case I: $N_{1}$ is connected, and, for every irreducible component $D$ of $N_{1}$, not all sections of $M_{1}$ vanish on $D$.

In this case, $M_{1}^{\otimes 2}=\omega_{N_{1}}, M_{1}$ has degree at most 5 , and, by Lemma 3.11 $\operatorname{deg} M_{1}=5$ if and only if $N_{1}=C$. By Proposition 3.4 $h^{0}\left(M_{1}\right) \leq 3$. Moreover, if equality holds, either $\operatorname{deg} M_{1}=5$ or $\operatorname{deg} M_{1}=4$ and $C$ is obtained from $N_{1}$ by identifying two points $p$ and $q$, which must then lie in the same component of $N_{1}$ by parity. Suppose that $\operatorname{deg} N_{1}=4$. Then $N_{1}$ does not have a separating node and thus, since equality holds in Clifford's theorem, $N_{1}$ has a nonsingular $g_{2}^{1}$. Since every irreducible component of $N_{1}$ meets the remaining components in at least 4 points, $N_{1}$ is stable and hyperelliptic. Thus $C$ satisfies (b) of [1, Theorem 4.10]. In this case, the Prym variety is a Jacobian, by the main theorem in [8] (where these curves are called quasi-trigonal), and hence $\operatorname{dim} \Xi_{\text {sing }} \geq 1$.

We may thus assume that $\operatorname{deg} M_{1}=5$, and hence that $N_{1}=C$. If $M_{1}$ has a base point which is a node, necessarily non-separating, then the 
proof of Proposition 3.4 shows that $h^{0}\left(M_{1}\right) \leq 2$, so that this case does not arise. If $M_{1}$ has a base point which is a smooth point of $C$, but no singular base point, then by Clifford's theorem $N_{1}=C$ has a nonsingular $g_{2}^{1}$ and so is hyperelliptic. Again, $\operatorname{dim} \Xi_{\text {sing }} \geq 1$. Otherwise $M_{1}$ defines a finite morphism $f: C \rightarrow \mathbb{P}^{2}$. If $f$ has positive degree on a component, or maps two components to the same irreducible curve in $\mathbb{P}^{2}$, then there exists a point $x \in f(C)$ such that $f^{-1}(x)=\left\{y_{1}, \ldots, y_{k}\right\}$ with $k \geq 2, x$ is a smooth point of $f(C)$, and each $y_{i}$ is a smooth point of $C$. It follows that $M_{1}\left(-\sum_{i} y_{i}\right)$ has degree at most three, is base point free, and $h^{0}\left(M_{1}\left(-\sum_{i} y_{i}\right)\right)=2$. The projection from $x$ defines a morphism $C \rightarrow \mathbb{P}^{1}$, which is finite by the arguments of Remark 3.3. Thus $C$ is either trigonal or hyperelliptic. By the main result of $[8], \operatorname{dim} \Xi_{\text {sing }} \geq 1$ in this case as well. In the remaining case, the morphism $f$ satisfies: $f_{*} \mathcal{O}_{C} / \mathcal{O}_{f(C)}$ is a skyscraper sheaf. Since $f(C)$ is a plane quintic, $p_{a}(C)=p_{a}(f(C))=6$, and hence $f_{*} \mathcal{O}_{C}=\mathcal{O}_{f(C)}$. It follows that $f$ is an embedding, $C$ is a plane quintic and $M_{1}$ is the pullback of $\mathcal{O}_{\mathbb{P}^{2}}(1)$.

Case II: $N_{1}$ is connected, but there is a component of $N_{1}$ on which all sections of $M_{1}$ vanish.

By Lemma 3.11 either $N_{1}$ is semistable or it is smooth rational, in which case $h^{0}\left(N_{1}\right)=0$. Assume that $N_{1}$ is semistable and let $\overline{N_{1}}$ be the associated stable curve, so that the theta characteristic $M_{1}$ is the pullback of a theta characteristic $\overline{M_{1}}$ on $\overline{N_{1}}$ with $h^{0}\left(\overline{M_{1}}\right)=h^{0}\left(M_{1}\right)$. Note that $\overline{M_{1}}$ has strictly positive degree on every component of $\overline{N_{1}}$. Clearly $\overline{N_{1}}$ and $\overline{M_{1}}$ satisfy the conclusions of Lemma 3.11. If the only components of $N_{1}$ where all sections of $M_{1}$ vanish are contained in fibers of the morphism $N_{1} \rightarrow \overline{N_{1}}$, then, for every irreducible component $\bar{D}$ of $\overline{N_{1}}$, not all sections of $\overline{M_{1}}$ vanish on $\bar{D}$, but there is a node of $\overline{N_{1}}$, the image of a component where all sections of $M_{1}$ vanish, which is in the base locus of $\overline{M_{1}}$. It follows as in Case I that $h^{0}\left(\overline{M_{1}}\right) \leq 2$. Thus, we may assume that there is a component of $\overline{N_{1}}$ where all sections of $\overline{M_{1}}$ vanish. Write $\overline{N_{1}}=\bar{N}^{\prime} \cup \bar{N}^{\prime \prime}$, where $\bar{N}^{\prime} \neq \emptyset$ is the union of all components of $\bar{N}_{1}$ on which all sections of $\overline{M_{1}}$ vanish. Note that $\#\left(\bar{N}^{\prime} \cap \bar{N}^{\prime \prime}\right)$ is even and $\operatorname{deg}\left(\overline{M_{1}} \mid \bar{N}^{\prime \prime}\right)=\operatorname{deg} \overline{M_{1}}-\operatorname{deg}\left(\overline{M_{1}} \mid \bar{N}^{\prime}\right) \leq 5-1=4$. If $\bar{N}^{\prime} \cap \bar{N}^{\prime \prime}=\left\{x_{1}, \ldots, x_{\ell}\right\}$, where the $x_{i}$ are distinct and $\ell=\#\left(\bar{N}^{\prime} \cap \bar{N}^{\prime \prime}\right)$, then $h^{0}\left(\overline{M_{1}}\right)=h^{0}\left(\left(\overline{M_{1}} \mid \overline{N^{\prime \prime}}\right)\left(-\sum_{i} x_{i}\right)\right)$, where

$$
0 \leq \operatorname{deg}\left(\overline{M_{1}} \mid \bar{N}^{\prime \prime}\right)\left(-\sum_{i} x_{i}\right)=\operatorname{deg}\left(\overline{M_{1}} \mid \bar{N}^{\prime \prime}\right)-\ell .
$$

Thus $\ell \leq 4$, so that $\ell$ is either 2 or 4 . If $\ell=\#\left(\bar{N}^{\prime} \cap \bar{N}^{\prime \prime}\right)=4$, then $\operatorname{deg}\left(\overline{M_{1}} \mid \bar{N}^{\prime \prime}\right)\left(-\sum_{i} x_{i}\right) \leq 0$. Since each connected component of $\bar{N}^{\prime \prime}$ meets 
the rest of $\overline{N_{1}}$ in at least two points, there are at most two connected components of $\bar{N}^{\prime \prime}$ and so $h^{0}\left(\overline{M_{1}}\right)=h^{0}\left(\left(\overline{M_{1}} \mid \bar{N}^{\prime \prime}\right)\left(-\sum_{i} x_{i}\right)\right) \leq 2$. If $\ell=2$, then $\bar{N}^{\prime \prime}$ is connected and the morphism $\bar{N}^{\prime \prime} \rightarrow C$ must have involved a normalization of $C$ at at least two nodes. In this case, $\operatorname{deg}\left(\overline{M_{1}} \mid \bar{N}^{\prime \prime}\right) \leq 3$, $\operatorname{deg}\left(\overline{M_{1}} \mid \bar{N}^{\prime \prime}\right)\left(-\sum_{i} x_{i}\right) \leq 1$, and hence

$$
h^{0}\left(\overline{M_{1}}\right)=h^{0}\left(\left(\overline{M_{1}} \mid \bar{N}^{\prime \prime}\right)\left(-\sum_{i} x_{i}\right)\right) \leq 2 .
$$

Hence neither case arises if $n_{1}$ is 3 or 4 .

Case III: $N_{1}$ is not connected.

Let $D_{1}, \ldots, D_{t}$ be the connected components of $N_{1}$ and let $C_{i}$ be the subscheme of $\widetilde{C}$ corresponding to $D_{i}$. If $d_{i}=\frac{1}{2} \operatorname{deg}\left(L \mid C_{i}\right)$, then $d_{i}>0$ and $\sum_{i} d_{i}=5$. By Lemma 3.11 $D_{i}$ is semistable or $D_{i}$ is smooth rational, and $\operatorname{deg}\left(M_{1} \mid D_{i}\right) \leq d_{i}-2$ since the image of $D_{i}$ in $C$ must meet the remaining components in at least 4 points. Moreover, $M_{1}$ is a theta characteristic on every component $D_{i}$. Finally, each irreducible component of $D_{i}$ meets the remaining components of $D_{i}$ in an even number of points. The possibilities for $\left(d_{1}, \ldots, d_{t}\right)$ are, after reordering, $(4,1),(3,2),(3,1,1),(2,2,1),(2,1,1,1)$, $(1,1,1,1,1)$, and thus $\operatorname{deg}\left(M_{1} \mid D_{i}\right) \leq 2$. If $\operatorname{deg}\left(M_{1} \mid D_{i}\right) \leq-1$, then $D_{i}$ is smooth rational and $h^{0}\left(M_{1} \mid D_{i}\right)=0$. If $\operatorname{deg}\left(M_{1} \mid D_{i}\right)=0$, then $D_{i}$ is semistable of arithmetic genus one, and $h^{0}\left(M_{1} \mid D_{i}\right) \leq 1$. If $\operatorname{deg}\left(M_{1} \mid D_{i}\right)=1$, then $d_{i} \geq 3, D_{i}$ is semistable of arithmetic genus two, there is exactly one irreducible component of $D_{i}$ such that $M_{1}$ restricts to a line bundle of degree one, and the remaining components are smooth rational curves meeting the rest of $D_{i}$ in two points. In particular, $M_{1} \mid D_{i}$ is induced by a line bundle $\overline{M_{1}}$ of degree one on the stable model $\overline{D_{i}}$ of $D_{i}$, which is irreducible of arithmetic genus two. It follows that $h^{0}\left(M_{1} \mid D_{i}\right)=h^{0}\left(\overline{M_{1}}\right) \leq 1$. If $D_{i}$ is a component such that $\operatorname{deg}\left(M_{1} \mid D_{i}\right)=2$, then $d_{i}=4$ and $M_{1} \mid D_{i}$ is induced by a line bundle $\overline{M_{1}}$ of degree two on the stable model $\overline{D_{i}}$ of $D_{i}$, which has arithmetic genus three and at most two components. If there exist sections of $\overline{M_{1}}$ which do not vanish on a component, then Proposition 3.4 implies that $h^{0}\left(M_{1} \mid D_{i}\right)=h^{0}\left(\overline{M_{1}}\right) \leq 2$. Otherwise, it is easy to see that $h^{0}\left(M_{1} \mid D_{i}\right)=h^{0}\left(\overline{M_{1}}\right)=0$. Running through the list of possible $\left(d_{1}, \ldots, d_{t}\right)$ above, it is easy to see that in all cases $h^{0}\left(M_{1}\right)=\sum_{i=1}^{t} h^{0}\left(M_{1} \mid D_{i}\right) \leq 2$.

Thus, examining all of the cases, we see that $h^{0}\left(M_{1}\right)=4$ is impossible, and if $h^{0}\left(M_{1}\right)=3$, then either $N_{1}=C$ and $M_{1}$ embeds $C$ as a plane quintic, or $C$ is trigonal or hyperelliptic or is obtained from a hyperelliptic curve by identifying two points. Thus, if $C$ is not a plane quintic, then $\operatorname{dim} \Xi_{\operatorname{sing}} \geq 1$. This concludes the proof of Theorem 3.13. 


\section{The proof of Theorem 2}

\subsection{Plane quintics and cubic threefolds}

Let $C$ be a reduced nodal curve, with normalization $\nu: N \rightarrow C$, and let $M$ be a theta characteristic on $N$. If the preimages of the nodes of $C$ are $p_{1}, q_{1}, \ldots, p_{\delta}, q_{\delta}$, then a local calculation shows that

$$
\begin{aligned}
\operatorname{Hom}\left(\nu_{*} M, \omega_{C}\right) & =\nu_{*}\left(M^{-1} \otimes \mathcal{O}_{N}\left(-\sum_{i}\left(p_{i}+q_{i}\right)\right) \otimes \nu^{*} \omega_{C}\right) \\
& =\nu_{*}\left(M^{-1} \otimes K_{N}\right)=\nu_{*} M .
\end{aligned}
$$

Using this, we have the following result of Beauville [3, Prop. 4.2] (cf. also [2, 6.27] as well as [5, Proposition V.2.3]):

Theorem 4.1. Let $C$ be a nodal plane quintic curve and $M$ a theta characteristic on the normalization $N$ of $C$ such that $h^{0}(M)=1$. Then there exists a symmetric matrix

$$
A=\left(\begin{array}{lll}
\ell_{1} & \ell_{2} & q_{1} \\
\ell_{2} & \ell_{3} & q_{2} \\
q_{1} & q_{2} & f
\end{array}\right),
$$

where the $\ell_{i}$ are linear polynomials in $x, y, z$, the $q_{i}$ are quadratic, and $f$ is cubic, such that the following is exact:

$$
0 \rightarrow \mathcal{O}_{\mathbb{P}^{2}}(-2)^{2} \oplus \mathcal{O}_{\mathbb{P}^{2}}(-3) \stackrel{A}{\rightarrow} \mathcal{O}_{\mathbb{P}^{2}}(-1)^{2} \oplus \mathcal{O}_{\mathbb{P}^{2}} \rightarrow \nu_{*} M \rightarrow 0 .
$$

In particular $\operatorname{det} A=\Delta$ is a polynomial of degree 5 defining $C$.

With hypotheses as in Theorem 4.1, let $x, y, z, w, t$ be homogeneous coordinates on $\mathbb{P}^{4}$ and consider the cubic threefold $X$ defined by the homogeneous cubic polynomial

$$
F(x, y, z, w, t)=\ell_{1} w^{2}+2 \ell_{2} w t+\ell_{3} t^{2}+2 q_{1} w+2 q_{2} t+f .
$$

Proposition 4.2. The cubic threefold $X$ contains the line $\lambda$ defined by $x=$ $y=z=0$. Under the assumption that $C$ is nodal and that $M$ is a theta characteristic on $N$ with $h^{0}(M)=1$, the threefold $X$ is smooth. Finally, the curve $C$ together with the theta characteristic $M$ on the normalization $N$ is the discriminant curve of the pair $(X, \lambda)$. 
Proof. Clearly $X$ contains $\lambda$. To see that $X$ is smooth, assume that $p_{0}$ is a point of Sing $X$. We first consider the case $p_{0}=\left(x_{0}, y_{0}, z_{0}, w_{0}, t_{0}\right)$ with $\left(x_{0}, y_{0}, z_{0}\right) \neq(0,0,0)$, i.e. $p_{0} \notin \lambda$. After a change of coordinates fixing $x, y, z$, we may assume that $\left(w_{0}, t_{0}\right)=(0,0)$. Since $p_{0}$ is a singular point of $X$,

$$
f\left(x_{0}, y_{0}, z_{0}\right)=q_{1}\left(x_{0}, y_{0}, z_{0}\right)=q_{2}\left(x_{0}, y_{0}, z_{0}\right)=0
$$

and $\left(x_{0}, y_{0}, z_{0}\right)$ is a singular point of $f$. In particular, the matrix $A$ is not invertible at $\left(x_{0}, y_{0}, z_{0}\right)$. Let $R$ be the local ring of $\mathbb{P}^{2}$ at $\left(x_{0}, y_{0}, z_{0}\right)$, with maximal ideal $\mathfrak{m}$, and continue to denote by $A$ the matrix with coefficients in $R$ induced by $A$. First assume that the matrix $A \bmod \mathfrak{m}$ has rank two at $\left(x_{0}, y_{0}, z_{0}\right)$. Then it is easy to check that $R^{3} / A \cdot R^{3} \cong R / \Delta$. After a linear change of coordinates in $w, t$, we may assume that $A \bmod \mathfrak{m}$ is of the form $\left(\begin{array}{lll}1 & 0 & 0 \\ 0 & 1 & 0 \\ 0 & 0 & 0\end{array}\right)$. Thus, $\Delta \equiv f \bmod \mathfrak{m}^{2}$. Since $\left(x_{0}, y_{0}, z_{0}\right)$ is a singular point of the cubic defined by $f=0, \Delta$ has a singular point at $\left(x_{0}, y_{0}, z_{0}\right)$, and the cokernel of $A$ is locally of the form $\mathcal{O}_{C}$, contradicting the hypotheses on $A$.

Next suppose that $A \bmod \mathfrak{m}$ has rank one. Then we may assume that $A \bmod \mathfrak{m}$ is of the form $\left(\begin{array}{lll}1 & 0 & 0 \\ 0 & 0 & 0 \\ 0 & 0 & 0\end{array}\right)$. Note that $\Delta \equiv \ell_{3} f-q_{2}^{2} \bmod \mathfrak{m}^{3}$. Since $f$ is singular at $\left(x_{0}, y_{0}, z_{0}\right), \ell_{3} f-q_{2}^{2} \equiv-q_{2}^{2} \bmod \mathfrak{m}^{3}$, and hence the tangent cone of $\ell_{3} f-q_{2}^{2}$ is degenerate. This contradicts the hypothesis on $\Delta$.

The remaining case is where the point $p_{0}$ lies on $\lambda$, in which case we may assume that $p_{0}=(0,0,0,1,0)$. Then $p_{0}$ is a singular point of $X$ if and only if $\ell_{1}=0$. In this case, $\Delta=-\left(q_{1}^{2} \ell_{3}-2 \ell_{2} q_{1} q_{2}+\ell_{2}^{2} f\right)$ automatically has a singular point at $\left(x_{0}, y_{0}, z_{0}\right)$ lying in the intersection of $\ell_{2}=q_{1}=0$. At such a point, if $\ell_{3} f-q_{2}^{2} \neq 0$, then the matrix $\left(\begin{array}{cc}\ell_{3} & q_{2} \\ q_{2} & f\end{array}\right)$ is nonsingular, and it is easy to see that $R^{3} / A \cdot R^{3} \cong R / \Delta$, a contradiction. Otherwise, it is easy to check that $\Delta$ is congruent to a square $\bmod \mathfrak{m}^{3}$, and so cannot have a nondegenerate tangent cone. For example, if there exists a $c$ such that $\ell_{3} \equiv c q_{2} \bmod \mathfrak{m}$ and $q_{2} \equiv c f \bmod \mathfrak{m}$, then

$$
\Delta \equiv-f\left(c q_{1}-\ell_{2}\right)^{2} \quad \bmod \mathfrak{m}^{3} .
$$

Once again, we reach a contradiction.

The final point to check is that the theta characteristic $M$ arises from the conic bundle structure on $X$. Since $X$ is nonsingular, $h=\ell_{1} \ell_{3}-\ell_{2}^{2}$ 
is nonzero. First assume that both $C$ and the conic $Q$ defined by $h$ are smooth. We claim that $Q$ meets $C$ everywhere with even multiplicity, and the support of the intersection is exactly the support of the sheaf $\nu_{*} M / \mathcal{O}_{C}$, where the inclusion $\mathcal{O}_{C} \rightarrow \nu_{*} M$ is defined by the unique section of $\nu_{*} M$. Note that

$\operatorname{det} A=f h+q_{1}\left(\ell_{2} q_{2}-q_{1} \ell_{3}\right)-q_{2}\left(\ell_{1} q_{2}-\ell_{2} q_{1}\right)=f h+\left(\ell_{3} q_{1}^{2}-2 \ell_{1} q_{1} q_{2}+\ell_{1} q_{2}^{2}\right)$,

and hence, if $p \in \mathbb{P}^{2}$ and $R=\mathcal{O}_{\mathbb{P}^{2}, p}$,

$$
R /(\operatorname{det} A, h)=R /\left(h, \ell_{3} q_{1}^{2}-2 \ell_{1} q_{1} q_{2}+\ell_{1} q_{2}^{2}\right) .
$$

Since $Q$ is smooth, at least one of $\ell_{1}, \ell_{3}$ is nonzero at $p$. If for example $\ell_{3} \neq 0$, then

$$
\ell_{3} q_{1}^{2}-2 \ell_{1} q_{1} q_{2}+\ell_{1} q_{2}^{2}=\frac{1}{\ell_{3}}\left(\ell_{3} q_{1}-\ell_{2} q_{2}\right)^{2} .
$$

The defining exact sequence for $\nu_{*} M$ shows that, at $p$,

$$
\left(\nu_{*} M / \mathcal{O}_{C}\right)_{p} \cong R^{2} /\left(\left(\ell_{1}, \ell_{2}\right),\left(\ell_{2}, \ell_{3}\right),\left(q_{1}, q_{2}\right)\right) .
$$

The homomorphism $R^{2} \rightarrow R / h$ defined by $(a, b) \mapsto a \ell_{3}-b \ell_{2}$ then defines an isomorphism from $\nu_{*} M / \mathcal{O}_{C}$ to $R /\left(h, \ell_{3} q_{1}-\ell_{2} q_{2}\right)$. Thus $\nu_{*} M / \mathcal{O}_{C}$ has support at $p$ if and only if $p \in C \cap Q$, and the length of the support there is one-half the local intersection number. It follows that the function field of $\widetilde{C}$ is generated by $\sqrt{\ell_{1} \ell_{3}-\ell_{2}^{2}}$. By [2, Lemma 1.6], the same holds for the double cover of $C$ defined by the discriminant curve construction. Hence the two double covers agree. This completes the proof when $C$ and $Q$ are smooth. For the general case, choose a pencil of cubic threefolds $X_{t}$ containing the line $\lambda$, specializing to the given pair $(X, \lambda)$, and such that, for the general member of the pencil, the discriminant curve and the conic defined by the first $2 \times 2$ minor of the matrix $A_{t}$ are smooth. A limiting argument, using the fact that $\mathcal{R}_{6}$ is separated, then imples that the corresponding admissible double covers agree for all values of $t$.

\subsection{Completion of the proof}

Let $A$ be a principally polarized abelian variety of dimension 5 . Then $A \cong P=P(\widetilde{C}, \pi)$ as principally polarized abelian varieties, where $\pi: \widetilde{C} \rightarrow C$ is an admissible double cover. If the theta divisor $\Xi$ of $P$ has a unique singular point which is a triple point, then, by Theorem 2.8 and Theorem 3.12 $C$ is a plane quintic and the theta characteristic $M$ on the normalization of 
$C$ corresponding to the double cover $\pi: \widetilde{C} \rightarrow C$ is odd. By Proposition 4.2 there exists a smooth cubic threefold $X$ and a line $\lambda$ on $X$ such that the discriminant curve of the pair $(X, \lambda)$ is $C$ and the corresponding theta characteristic on $N$ is $M$. By Beauville's result [2, Proposition 2.8], $J X \cong P$ as principally polarized abelian varieties, and hence $A \cong J X$. This concludes the proof of Theorem 2 .

\section{References}

[1] A. Beauville, Prym varieties and the Schottky problem, Invent. Math. 41 (1977), 149-196.

[2] A. Beauville, Variétés de Prym et Jacobiennes intermédiares, Ann. scient. Éc. Norm. Sup. 10 (1977), 309-391.

[3] A. Beauville, Determinantal hypersurfaces, Michigan Math. J. 48 (2000), $39-64$.

[4] C. H. Clemens and P. Griffiths, The intermediate Jacobian of the cubic threefold, Annals of Math. 95 (1972), 281-356.

[5] R. Donagi and R. Smith, The structure of the Prym map, Acta Math. 146 (1981), 26-102.

[6] D. Mumford, Abelian varieties, Bombay, Oxford University Press, 1970.

[7] D. Mumford, Prym varieties I, in Contributions to Analysis (a collection of papers dedicated to Lipman Bers), 325-350, New York, Academic Press, 1974.

[8] V. V. Shokurov, Prym varieties: theory and applications, Math USSR Izv. 23 (1984), 83-147.

[9] R. Smith and R. Varley, A Riemann singularities theorem for Prym theta divisors, with applications, preprint.

Department of Mathematics

Columbia University

New York, NY 10027

USA

casa@math.columbia.edu, rf@math.columbia.edu 